\title{
Empirical distributions of daily equity index returns: A comparison
}

\author{
Canan G. Corlua ${ }^{\mathrm{a}}$, Melike Meterelliyoz ${ }^{\mathrm{b}, *}$, Murat Tiniç ${ }^{\mathrm{c}}$ \\ a Metropolitan College, Boston University, Boston, MA 02215 USA \\ ${ }^{\mathrm{b}}$ Department of Business Administration, TOBB University of Economics and Technology, Ankara 06560 Turkey \\ ${ }^{\mathrm{c}}$ Department of Management, Bilkent University, Ankara 06800 Turkey
}

\section{A R T I C L E I N F O}

\section{Keywords:}

Index returns

Generalized lambda

Johnson translation system

Skewed- $t$

Normal inverse Gaussian

g-and-h

\begin{abstract}
A B S T R A C T
The normality assumption concerning the distribution of equity returns has long been challenged both empirically and theoretically. Alternative distributions have been proposed to better capture the characteristics of equity return data. This paper investigates the ability of five alternative distributions to represent the behavior of daily equity index returns over the period 1979-2014: the skewed Student- $t$ distribution, the generalized lambda distribution, the Johnson system of distributions, the normal inverse Gaussian distribution, and the g-and-h distribution. We find that the generalized lambda distribution is a prominent alternative for modeling the behavior of daily equity index returns.
\end{abstract}

(C) 2016 Elsevier Ltd. All rights reserved.

\section{Introduction}

The assumption that stock price changes follow a stable distribution forms the basis for major asset pricing and option pricing models. Early models by Bachelier (1900) take normality as a fundamental assumption for modeling stock price movements. In line with this assumption, Osborne (1959) shows that logarithms of the changes in the stock prices are mutually independent with a common probability distribution (i.e., they conform to a random walk). He then suggests that stock price changes must follow a normal distribution. However, these findings have been challenged both theoretically and empirically. ${ }^{1}$

An early work by Mandelbrot (1967) proposes that stock price returns belong to the family of stable Paretian distributions because they have fatter tails. Fama $(1963 ; 1965)$ provides empirical evidence that supports this claim and demonstrates that stock price changes indeed have fatter tails and have higher peaks than the normal distribution. More recently, Rachev, Stoyanov, Biglova, and Fabozzi (2005) compared the stable Paretian distribution to the normal distribution using 382 US stock returns over the period 1992-2003. The authors investigated the daily returns using two probability models: the homoskedastic independent and identically distributed model and the conditional heteroskedastic ARMA-GARCH model. Normality was rejected for both models. However, Officer (1972) found that normality holds for monthly

\footnotetext{
* Corresponding author. Tel.: +90 3122924214

E-mail addresses: canan@bu.edu (C.G. Corlu), mkuyzu@etu.edu.tr (M. Meterelliyoz), tinic@bilkent.edu.tr (M. Tiniç).

${ }^{1}$ A similar line of argument holds for exchange rate returns.
}

returns and that the standard deviation of the returns is inconsistent with the stable hypothesis. To support this argument, Praetz (1972) then suggested the Student- $t$ distribution as an alternative to the stable Paretian because the stable Paretian distribution has an infinite variance property and the density function of the stable Paretian is unknown. Over an eight-year period, Praetz (1972) examined weekly data from Sydney Stock Exchange and showed that the Student- $t$ distribution can be used as an alternative to explain the stock price behavior.

The Student- $t$ distribution was also compared with the normal distribution and the Cauchy distribution by Blattberg and Gonedes (1974). Contrary to Praetz (1972), they used both daily and weekly returns of stocks of the Dow Jones Industrial (DJI), and they used the maximum likelihood estimation method for estimating the parameters of the distributions. Blattberg and Gonedes (1974) showed that the Student- $t$ distribution performs better than the normal distribution on daily returns. However, normality is not rejected for monthly return data. Hagerman (1978) tested the normality hypothesis on both individual stocks of the American and New York Stock Exchanges on portfolios that contain these stocks, and found that they do not behave in line with the normal distribution. Hagerman (1978) proposed that the mixture of normal distributions and the Student- $t$ distribution can be an alternative to representing the characteristics of stock return data. However, the performance of these two distributions against each other was not investigated in Hagerman's work.

Kon (1984) compared the discrete mixture of normal distributions and the Student- $t$ distribution over a period of almost 19 years, examining daily returns of 30 stocks from DJI and Standard \& Poor's (S\&P) value- and equal-weighted stock market indexes. A 
discrete mixture of normal distributions is shown to have greater validity than the Student- $t$ distribution in modeling the data. Similar to Blattberg and Gonedes (1974) and Akgiray and Booth (1987); Officer (1972) stated that the monthly returns of stock prices can be assumed to be normally distributed. However, for the daily data they found that the mixed diffusion process and the mixture of normal distributions perform better than the stable distributions.

Bookstaber and McDonald (1987) proposed the generalized beta (GB2) distribution to explain the behavior of stock returns. This was chosen because the GB2 is a flexible distribution and acknowledges various distributions as special cases. They found that the GB2 distribution is significantly better than the lognormal distribution, especially in relation to short time intervals. Badrinath and Chatterjee (1988) examined the Center for Research in Security Prices (CRSP) value-weighted market index returns between 1962 and 1985 and concluded that returns of stock prices follow a skewed g-and-h distribution. ${ }^{2}$ Similarly, Mills (1995) found that the g-and-h distribution accurately fits a dataset that consists of three London Stock Exchange indices: FTSE 100, Mid 250, and FTSE 350.

A more general comparison of distributions with finite variances over equity stocks was conducted by Gray and French (1990). They compared the scaled- $t$ distribution, the logistic distribution, the exponential power distribution, and the normal distribution over the log-returns of daily S\&P 500 Composite index values for the period 1979-1985. Among four alternatives, the exponential power distribution was found to be the best fit. Lau, Lau, and Wingender (1990) showed that series of returns of stock prices that are taken from the CRSP yield higher kurtosis and skewness than the normal distribution. They proposed the lognormal, beta, Weibull, Pearson Types IV and VI, and Johnson system of distributions as alternatives. A general comparison of the normal distribution to the scaled- $t$ distribution and to the mixture of two normal distributions was conducted by Aparicio and Estrada (2001) using the daily returns of 13 different European stock markets. It was found that the scaled- $t$ distribution is a significantly better fit for the data, and the partial mixture of two normal distributions also performs well. Normality is rejected in all cases.

Linden (2001) introduced the Laplace mixture distribution, which is derived by conditioning the standard deviation of the normal distribution as an exponentially distributed random variable. Linden (2001) used this distribution to represent the daily, weekly, and monthly returns of the 20 most traded shares and the index of the Helsinki Stock Market. The normality assumption is not always rejected for the weekly and monthly returns. However, for the daily returns, an asymmetric Laplace distribution is found to be a better candidate than the normal distribution.

Harris and Küçüközmen (2001a) and Harris and Küçüközmen (2001b), respectively, examined the skewed generalized-t distribution (SGT) and the exponential generalized beta distribution (EGB) using daily UK, US, and Turkish equity returns. Consequently, they found that the SGT outperforms the EGB. In both studies, the authors rejected the hypothesis that the daily returns are distributed with the Student- $t$, power of exponential, or logistic distribution. In addition, for the daily Turkish returns, the Laplace distribution was also rejected. For the UK returns, the skewed- $t$ distribution was preferred, whereas for the US returns, the generalized-t distribution was preferred. More recently, Behr and Pötter (2009) compared the generalized hyperbolic distribution, the generalized $\log F$ distribution, and the finite mixture of Gaussians on monthly S\&P 500 index returns over the years 1871-2005 and daily returns over the years 2001-2005. For the monthly returns, the two-component

\footnotetext{
${ }^{2}$ Badrinath and Chatterjee (1988) also provide an excellent review of the literature.
}

Gaussian mixture distribution described the empirical distribution of the returns better than alternative distributions. Although the generalized hyperbolic distribution is the poorest performer for monthly returns, it performs best for daily data. However, as the daily data examined by Behr and Pötter (2009) is almost symmetric, the Laplace distribution, which does not have a parameter to capture the asymmetries, fits as well as the generalized hyperbolic distribution.

Finally, as an alternative to the stable distribution and the Student- $t$ distribution, Chalabi, Scott, and Würtz (2010) use the generalized lambda distribution (GLD) for modeling equity returns. Starting with Eberlein and Keller (1995), the normal inverse Gaussian (NIG) distribution is used to model financial returns and particularly for modeling 30 stocks at the German Stock Index. Prause, Zentrum, and Modellbildung (1997) show the applicability of the NIG distribution in modeling German stock and US Stock Index data. Bølviken and Benth (2000) used the NIG distribution to model 8 Norwegian stocks.

In Table 1, we summarize the papers that performed comparison studies to investigate the behavior of stock returns. We find that the outcomes differ and are often conflicting. Based upon this, our goal in this study is to fill this gap in the literature by addressing which distribution is best for modeling daily equity index return data. To this end, we consider the following flexible distributions that are commonly used in finance: the skewed Student$t$ distribution, the GLD, the NIG distribution, the Johnson system of distributions, and the g-and-h distribution. We conduct a comprehensive numerical analysis to compare the overall suitability of these five distributions on the equity index returns of twenty different countries over the period 1979-2014, which is divided into twelve three-year sub-periods. We also include the normal distribution in our experimental design. The overall suitability is initially compared using the Kolmogorov-Smirnov (KS) test statistic (Chakravarti \& Laha, 1967) and the Anderson-Darling (AD) test statistic (Anderson \& Darling, 1954). Furthermore, we conduct $p$ value tests in order to assess the significance of these KS and $\mathrm{AD}$ statistics. In addition, the explanatory power of the models is tested using in-sample Value-at-Risk (VaR) failure rates. Consistent with other studies in the previous research, we find that normality is rejected in all sub-periods for all markets. Our $p$-value tests and the in-sample VaR test suggest that GLD performs best for all markets over all time periods.

The remainder of the paper is organized as follows. Section 2 presents the data. Section 3 presents the distributions along with the fitting methods that are used to estimate the parameters of the distributions. Section 4 discusses our numerical study and Section 5 presents key conclusions.

\section{Description of the data}

We create a diversified sample from ten developed and ten emerging market indexes. The selected developed stock market indexes are: S\&P/ASX 200 Index (Australia), S\&P/Toronto Stock Exchange Index (Canada), CAC 40 (France), DAX (Germany), NIKKEI 225 (Japan), the Straits Times Index (Singapore), IBEX 35 (Spain), SMI (Switzerland), FTSE 100 (UK), and S\&P 500 (US), while the emerging stock market indexes are the Ibovespa Index (Brazil), IPSA Index (Chile), SHSZ 300 (China), BSE 500 (India), KOSPI Index (Korea), FBMKLCI Index (Malaysia), the Mexican IPC Index (Mexico), MICEX Index (Russia), JALSH Index (South Africa), and BIST 100 (Turkey). The daily closing index levels from January 1979 to August 2014 are collected using the Bloomberg Terminal.

Bloomberg provides index levels for the S\&P/ASX 200, CAC 40, DAX and IBEX 35 prior to their establishment date. This can happen due to two reasons. First, the index levels can be adjusted with respect to their ancestor indices. For instance, the DAX 
Table 1

Summary of articles that performed comparison studies to investigate the behavior of stock returns. The first column of this table lists the articles that compare several distributions for modeling stock returns. The second column of the table presents the distributions considered in the corresponding study.

\begin{tabular}{|c|c|}
\hline Article & Compared distributions \\
\hline Fama (1963), Fama (1965) and Rachev et al. (2005) & Stable Paretian*, normal \\
\hline Praetz (1972) & Scaled-t*, compound process, normal \\
\hline Hsu, Miller, and Wichern (1974) & Normal*, stable Paretian \\
\hline Blattberg and Gonedes (1974) & Student-t*, Cauchy, normal \\
\hline Kon (1984) & Discrete mixture of normals*, Student- $t$ \\
\hline Eberlein and Keller (1995) & Normal inverse Gaussian*, normal \\
\hline Akgiray and Booth (1987) & Mixed diffusion process*, discrete mixture of normals \\
\hline Bookstaber and McDonald (1987) & Generalized beta*, lognormal \\
\hline Gray and French (1990) & Exponential power*, logistic, Scaled-t, normal \\
\hline Aparicio and Estrada (2001) & Scaled-t*, mixture of normal, normal \\
\hline Linden (2001) & Asymmetric Laplace*, normal \\
\hline Harris and Küçüközmen (2001a), (2001b) & $S G T^{*}$, EGB, exponential power, logistic, Student- $t$, Laplace \\
\hline Behr and Pötter (2009) & Finite mixture of Gaussians*, generalized hyperbolic**, generalized logF \\
\hline
\end{tabular}

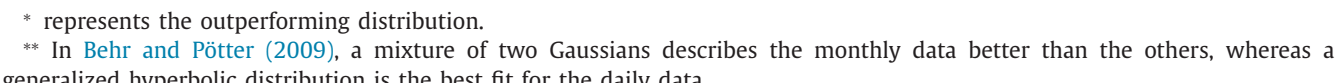
generalized hyperbolic distribution is the best fit for the daily data.

Table 2

Markets considered in each sub-period. This table presents the emerging and developed markets considered in each sub-period. For instance, in sub-period 1979-1981, data are available only for the developed markets Canada, Germany, Japan, and the US and the emerging markets Korea and Malaysia. Beginning from the sub-period 2000-2002, we have access to data for all ten developed and ten emerging markets.

\begin{tabular}{|c|c|c|}
\hline Periods & Developed markets & Emerging markets \\
\hline 1979-1981 & Canada, Germany, Japan, US & Korea, Malaysia \\
\hline 1982-1984 & Canada, Germany, Japan, UK, US & Korea, Malaysia \\
\hline 1985-1987 & Canada, France, Germany, Japan, Spain, UK, US & Korea, Malaysia \\
\hline $1988-1990$ & $\begin{array}{l}\text { Canada, France, Germany, Japan, } \\
\text { Spain, Switzerland, UK, US }\end{array}$ & Chile, Korea, Malaysia, Turkey \\
\hline 1991-1993 & $\begin{array}{l}\text { Australia, Canada, France, Germany } \\
\text { Japan, Spain, Switzerland, UK, US }\end{array}$ & Brazil, Chile, Korea, Malaysia, Turkey \\
\hline 1994-1996 & $\begin{array}{l}\text { Australia, Canada, France, Germany } \\
\text { Japan, Spain, Switzerland, UK, US }\end{array}$ & $\begin{array}{l}\text { Brazil, Chile, Korea, Malaysia } \\
\text { Mexico, South Africa, Turkey }\end{array}$ \\
\hline 1997-1999 & $\begin{array}{l}\text { Australia, Canada, France, Germany, Japan } \\
\text { Singapore, Spain, Switzerland, UK, US }\end{array}$ & $\begin{array}{l}\text { Brazil, Chile, India, Korea, Malaysia } \\
\text { Mexico, Russia, South Africa, Turkey }\end{array}$ \\
\hline $2000-2014$ & All markets & All markets \\
\hline
\end{tabular}

follows its ancestor indices Börsenzeitungs Index and Hardy Index. ${ }^{3}$ Second, the index levels can be recalculated to an earlier date. For instance, the CAC 40 is established in December 31, 1987; however, CAC 40 index levels are recalculated on a daily basis to September 7, 1987 (Shilling, 1996). Similarly, the S\&P/ASX 200 is established in April 3, 2000; however, S\&P/ASX 200 index levels are recalculated to May, $1992 .{ }^{4}$ IBEX 35 is launched in January 14, 1992 and IBEX 35 index levels are recalculated to January 5, 1987 (Fernandez, Aguirreamalloa, \& Avendaño, 2011).

The daily logarithmic returns are calculated as $X_{t}=$ $\log \left(S_{t} / S_{t-1}\right)$, where $S_{t}$ is the closing index level at time $t$. We divide the data for each market into twelve three-year nonoverlapping sub-periods. Our sample period contains both regional and global financial crises such as the 1997 Asian financial crisis, the Global Financial Crisis (2006-2008), and the Euro-zone Sovereign Debt Crisis (2009-2011). Unfortunately, return data is not available for every market during all of these sub-periods. In Table 2, we list data that are available for the developed and the emerging markets in each sub-period. For example, in sub-period 19791981, data are available only for the developed markets Canada, Germany, Japan, and the US and the emerging markets Korea and Malaysia. Beginning from the sub-period 2000-2002, we have access to data for all ten developed and ten emerging markets.

Fig. 1 presents the descriptive statistics for the developed markets and the emerging markets over different sub-periods. The

\footnotetext{
3 Based on communications with Deutsche Börse Group.

${ }^{4}$ Based on communications with Standard \& Poor's Dow Jones Indices.
}

number in each circle represents the sub-period number, where 1 is for the sub-period $1979-1981$ and 13 is for the entire sample. The skewness equals to $s=\left(m_{3} / m_{2}\right)^{3 / 2}$ and the kurtosis is given by $k=m_{4} / m_{2}^{2}$, where $m_{i}$ is the estimate of the $i$ th moment around the mean. The skewness values of the returns in the figure are clustered either on the right or left hand-side of the vertical line drawn at zero, indicating that all returns of all indices demonstrate a skewed (either left or right) behavior. Similarly, the kurtosis of returns are all clustered above the line drawn at three (some being very far from three) meaning that the return distributions are more peaked than the normal distribution.

For the developed markets in Fig. 1, the fourth sub-period (i.e., 1988-1990) and the thirteenth sub-period (i.e., the entire sample) exhibit skewness and kurtosis values that are significantly far from the skewness and kurtosis values of the normal distribution. For the emerging markets, there are more sub-periods that exhibit high skewness and kurtosis values, such as fourth, seventh, eighth, ninth, twelfth, and thirteenth sub-periods (i.e., 1988-1990, 19971999, 2000-2002, 2003-2005, 2012-2014, entire sample). These two figures alone indeed suggest that the normal distribution is not a good model for describing the distribution of daily equity index returns. Additionally, we calculate the Bera-Jarque (BJ) statistic for all of the countries over all sub-samples in order to test for any departure from normality. The results indicate that the normality is rejected in $96 \%$ of the instances, at the $1 \%$ significance level. ${ }^{5}$

\footnotetext{
${ }^{5}$ To save space, we do not present the BJ test statistics here. However, they are available upon request.
} 
Descriptive statistics for the developed markets

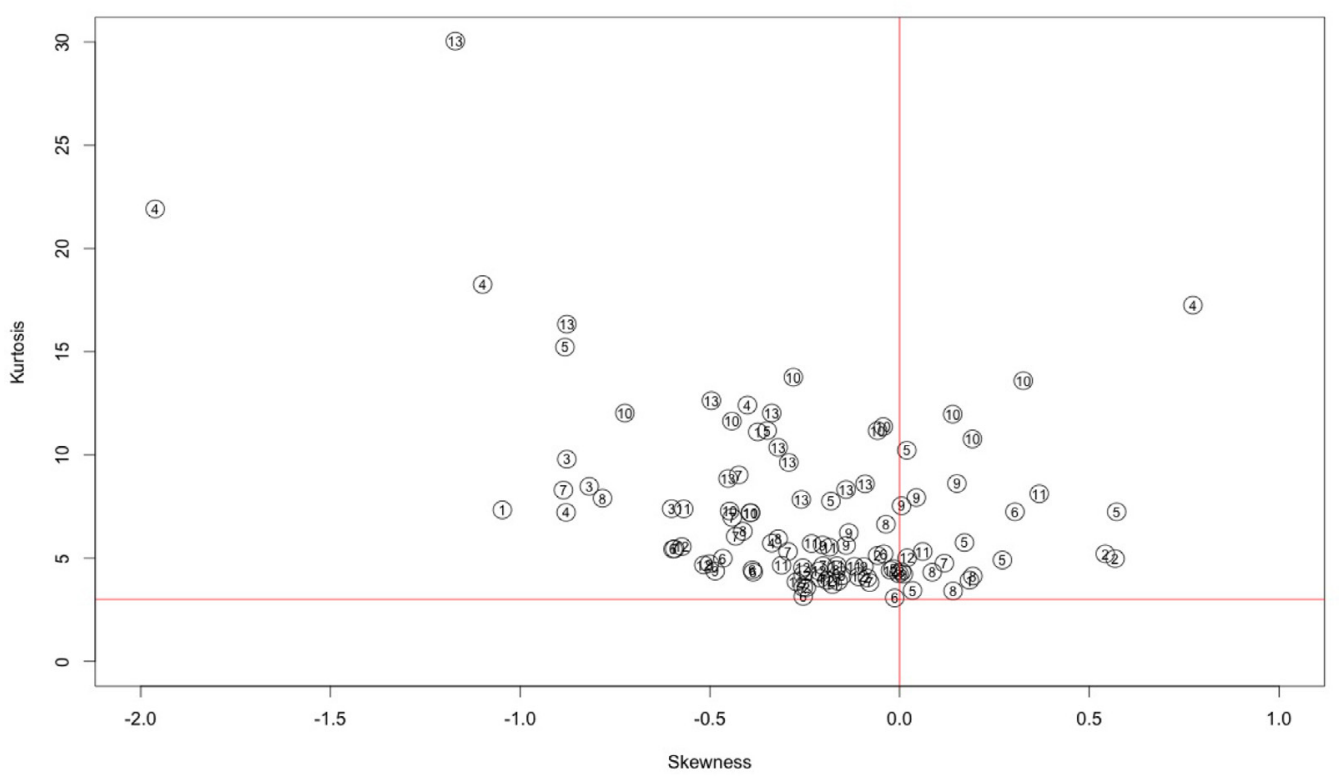

Descriptive statistics for the emerging markets

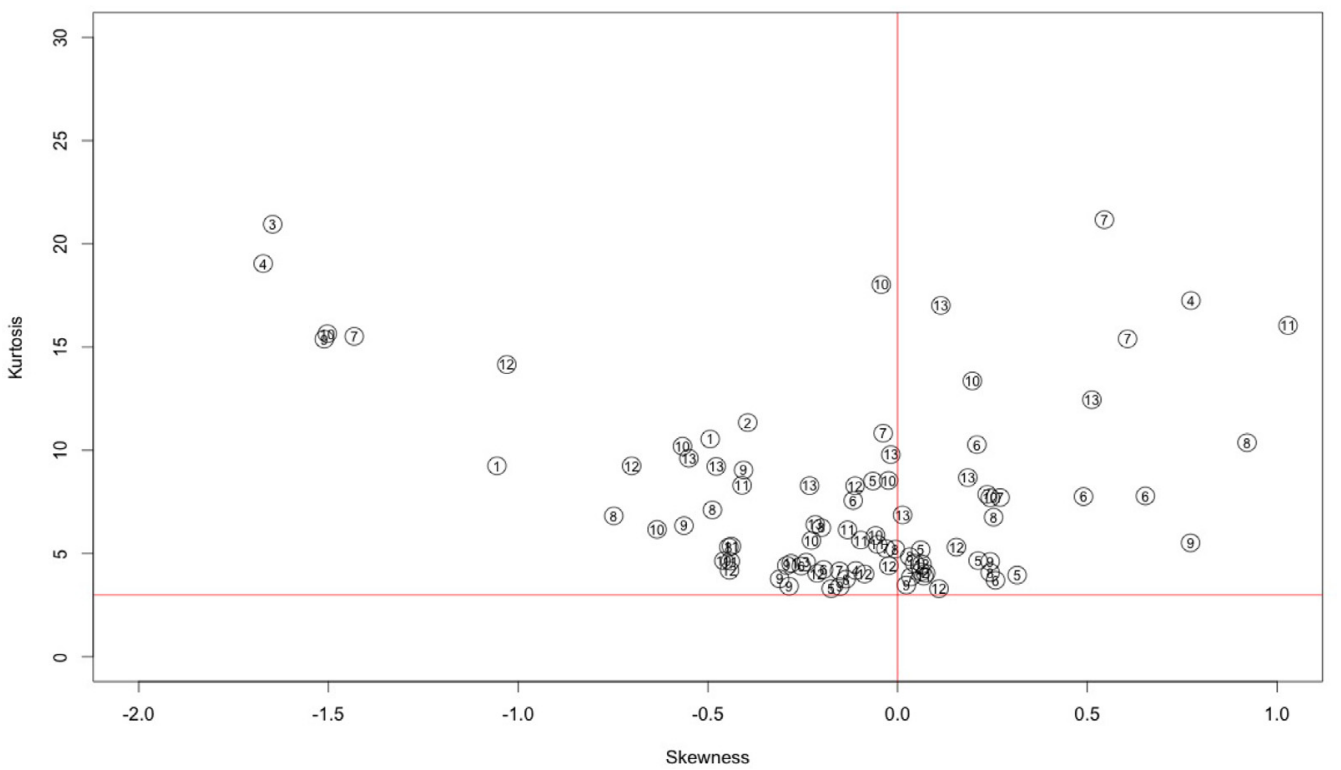

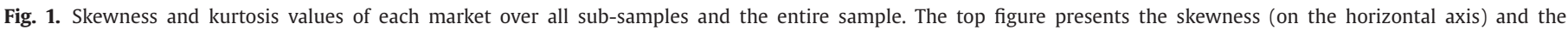

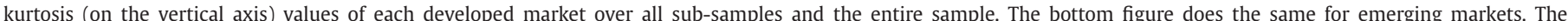

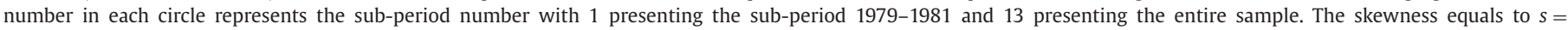
$\left(m_{3} / m_{2}\right)^{3 / 2}$ and the kurtosis is given by $k=m_{4} / m_{2}^{2}$, where $m_{i}$ is the estimate of the $i$ th moment around the mean.

\section{Flexible distributions}

We describe the generalized lambda distribution (GLD) in Section 3.1, the Johnson translation system in Section 3.2, the skewed Student- $t$ distribution in Section 3.3, the normal inverse Gaussian (NIG) distribution in Section 3.4, and the g-and-h distribution in Section 3.5.

\subsection{The Generalized Lambda distribution}

The GLD (Filliben, 1975; Joiner \& Rosenblatt, 1971; Ramberg \& Schmeiser, 1974), which is an extension of Tukey's lambda distribution (Hastings, Mosteller, Tukey, \& Winsor, 1947), is defined by the following inverse cumulative distribution function:

$F^{-1}\left(u ; \lambda_{1}, \lambda_{2}, \lambda_{3}, \lambda_{4}\right)=\lambda_{1}+\frac{u^{\lambda_{3}}-(1-u)^{\lambda_{4}}}{\lambda_{2}}$,

where $0 \leq u \leq 1 ; \lambda_{1}$ is the location parameter, $\lambda_{2}$ is the scale parameter, and $\lambda_{3}$ and $\lambda_{4}$ are related to skewness and kurtosis, respectively. This representation is denoted as Ramberg-Schmeiser Generalized Lambda Distribution (RS GLD) in reference to the parametrization of Ramberg and Schmeiser (1974). However, the probability density function (pdf) associated with equation (3.1) does not provide a valid pdf for all combinations of $\lambda_{3}$ and $\lambda_{4}$ (Fournier et al., 2007).

In order to avoid this problem, Freimer, Kollia, Mudholkar, and Lin (1988) proposed a different parametrization of the GLD, 
denoted as Freimer-Mudholkar-Kollia-Lin Generalized Lambda Distribution (FMKL GLD). This is given by:

$F^{-1}\left(u ; \lambda_{1}, \lambda_{2}, \lambda_{3}, \lambda_{4}\right)=\lambda_{1}+\frac{1}{\lambda_{2}}\left(\frac{u^{\lambda_{3}}-1}{\lambda_{3}}-\frac{(1-u)^{\lambda_{4}}-1}{\lambda_{4}}\right)$.

Both the FMKL and RS representations are used in practice as they offer a wide variety of shapes. However, the FMKL representation is preferable as a result of its ease of use. In addition, it is well defined over all parameter values with the only restriction being that $\lambda_{2}$ must be positive. Also, the condition $\min \left(\lambda_{3}, \lambda_{4}\right)>-1 / k$ must hold to have a finite $k$ th moment. In this study, we use the FMKL representation. The FMKL GLD curves are classified into five categories: Class-I family $\left(\lambda_{3}<1, \lambda_{4}<1\right)$ represents unimodal densities with continuous tails, Class-II family $\left(\lambda_{3}>1, \lambda_{4}<1\right)$ represents monotone pdfs similar to the exponential distribution, Class-III family ( $1<\lambda_{3}<2,1<\lambda_{4}<2$ ) represents U-shaped densities with truncated tails, Class-IV family $\left(\lambda_{3}>2,1<\lambda_{4}<\right.$ 2) represents $S$-shaped densities, and finally Class-V family $\left(\lambda_{3}>\right.$ $2, \lambda_{4}>2$ ) represents unimodal densities with truncated tails. We find that the daily equity return data belongs to the Class-I family.

Prior studies propose different fitting techniques for estimating the parameters of the GLD. ${ }^{6}$ In this study, we use the maximum likelihood estimation method in the GLDEX package of $\mathrm{R}$ (Su, 2007).

\subsection{The Johnson translation system}

A random variable $\mathrm{X}$ from the Johnson translation system is represented by (Johnson, 1949):

$X=\xi+\lambda r^{-1}\left(\frac{Z-\gamma}{\delta}\right)$,

where $\mathrm{Z}$ is a standard normal random variable, $\gamma$ and $\delta$ are shape parameters, $\xi$ is a location parameter, $\lambda$ is a scale parameter, and $r(\cdot)$ is one of the following transformations:

$r(X)=\left\{\begin{array}{l}x \\ \log (x) \\ \log (x /(1-x)) \\ \log \left(x+\sqrt{x^{2}+1}\right)\end{array}\right.$

for the $S_{N}$ (normal) family for the $S_{L}$ (lognormal) family for the $S_{B}$ (bounded) family for the $S_{U}$ (unbounded) family.

The range of the random variable $X$ is defined by the family of interest: $X>\xi$ and $\lambda=1$ for the $S_{L}$ family; $\xi<X<\xi+\lambda$ for the $S_{B}$ family; and $-\infty<X<\infty$ for the $S_{N}$ and $S_{U}$ families. For each feasible combination of the skewness and the kurtosis values there is a unique family that depends on the choice of $r$. In this study, we only consider the $S_{U}$ family of the Johnson translation system because the equity index returns have skewness and kurtosis values that conform with the $S_{U}$ family.

There are alternative methodologies proposed in previous research to estimate the parameters of the $S_{U}{ }^{7}$ We use the methodology proposed in Tuenter (2001), which resembles the method of moments, where the sample skewness and kurtosis are equalized with the theoretical skewness and kurtosis.

\subsection{The skewed Student-t distribution}

A number of skewed Student- $t$ distributions have been proposed in previous research. In this study, due to its simplicity, we follow the parametrization used in Azzalini and Capitanio (2003).

\footnotetext{
${ }^{6}$ A detailed review can be found in Corlu and Meterelliyoz (2015).

7 A detailed review can be found in Corlu and Biller (2015).
}

A random variable $\mathrm{X}$ from the skewed Student- $t$ distribution (hereafter, skewed- $t$ distribution) has a density of the form:

$f(x ; \delta, v, \mu, \beta)=\frac{1}{\delta} t_{v}\left(\frac{x-\mu}{\delta}\right) 2 T_{v+1}\left(\beta\left(\frac{x-\mu}{\delta}\right) \sqrt{\frac{v+1}{\left(\frac{x-\mu}{\delta}\right)^{2}+v}}\right)$,

where $\mu, \delta$, and $\beta$ represent the location, scale, and skewness parameters, respectively. $t_{v}$ is the density of the standard Student- $t$ distribution with $v$ degrees of freedom and $T_{v+1}$ is the distribution function of the standard Student- $t$ distribution with $v+1$ degrees of freedom.

Estimates of the parameters of the skewed- $t$ distribution are obtained using the maximum likelihood estimation method proposed by Azzalini and Capitanio (2003). The maximization of the likelihood function is conducted by the Nelder-Mead algorithm.

\subsection{The Normal Inverse Gaussian distribution}

The NIG distribution is obtained from a more general distribution called the generalized hyperbolic distribution (GHD), whose density is given by:

$$
\begin{aligned}
f(x ; \lambda, \alpha, \beta, \mu, \delta) & \\
= & \frac{\left(\delta \sqrt{\alpha^{2}-\beta^{2}}\right)^{\lambda}(\delta \alpha)^{1 / 2-\lambda}}{\sqrt{2 \pi} \delta K_{\lambda}\left(\delta \sqrt{\alpha^{2}-\beta^{2}}\right)}\left(1+\frac{(x-\mu)^{2}}{\delta^{2}}\right)^{\lambda / 2-1 / 4} \\
& \times \exp (\beta(x-\mu)) K_{\lambda-1 / 2}\left(\alpha \delta \sqrt{1+\frac{(x-\mu)^{2}}{\delta^{2}}}\right),
\end{aligned}
$$

where $K_{\lambda}$ is the modified third-order Bessel function; $\mu$ and $\delta$ represent location and scale parameters, respectively; $\lambda$ is the classdefining parameter; $\alpha$ is a parameter related to tail heaviness; and $\beta$ is the asymmetry parameter. The density is defined under the following parameter restrictions:

$$
\begin{aligned}
& \delta \geq 0 \text { and }|\beta|<\alpha \text { if } \lambda>0 \\
& \delta>0 \text { and }|\beta|<\alpha \text { if } \lambda=0 \\
& \delta>0 \text { and }|\beta| \leq \alpha \text { if } \lambda<0
\end{aligned}
$$

Pfaff, McNeil, and Ulmann (2013) indicate that the GHD can represent skewed distributions as well as heavy tails. The variants of the GHD can be obtained by changing the value of the parameter $\lambda$; this is why $\lambda$ is called the class-defining parameter. The NIG distribution is obtained from the GHD by setting $\lambda=-1 / 2$, and its density is given by:

$f(x ; \alpha, \beta, \mu, \delta)=\frac{\alpha \delta K_{1}\left(\alpha \sqrt{\delta^{2}+(x-\mu)^{2}}\right)}{\pi \sqrt{\delta^{2}+(x-\mu)^{2}}} e^{\delta \sqrt{\alpha^{2}-\beta^{2}}+\beta(x-\mu)}$,

with $|\beta| \leq \alpha$ and $\delta>0$ (Prause, 1999).

We use the ghyp package of $\mathrm{R}$ to estimate the parameters of the NIG.

\subsection{The g-and-h distribution}

The g-and-h distribution is a functional transformation of the standard normal distribution. A random variable from the g-and-h distribution is obtained by transforming the standard normal random variable $Z$ to the following form (Tukey, 1977):

$Y_{g, h}(Z)=\left(e^{g Z}-1\right) \frac{\exp \left(h Z^{2} / 2\right)}{g}$,

where $g, h \in \mathbb{R}$. The $g$ and $h$ parameters account for skewness and kurtosis, respectively. When a location parameter $A$ and a scale parameter $B$ are incorporated, a random variable from the g-and-h 


\section{Canada}
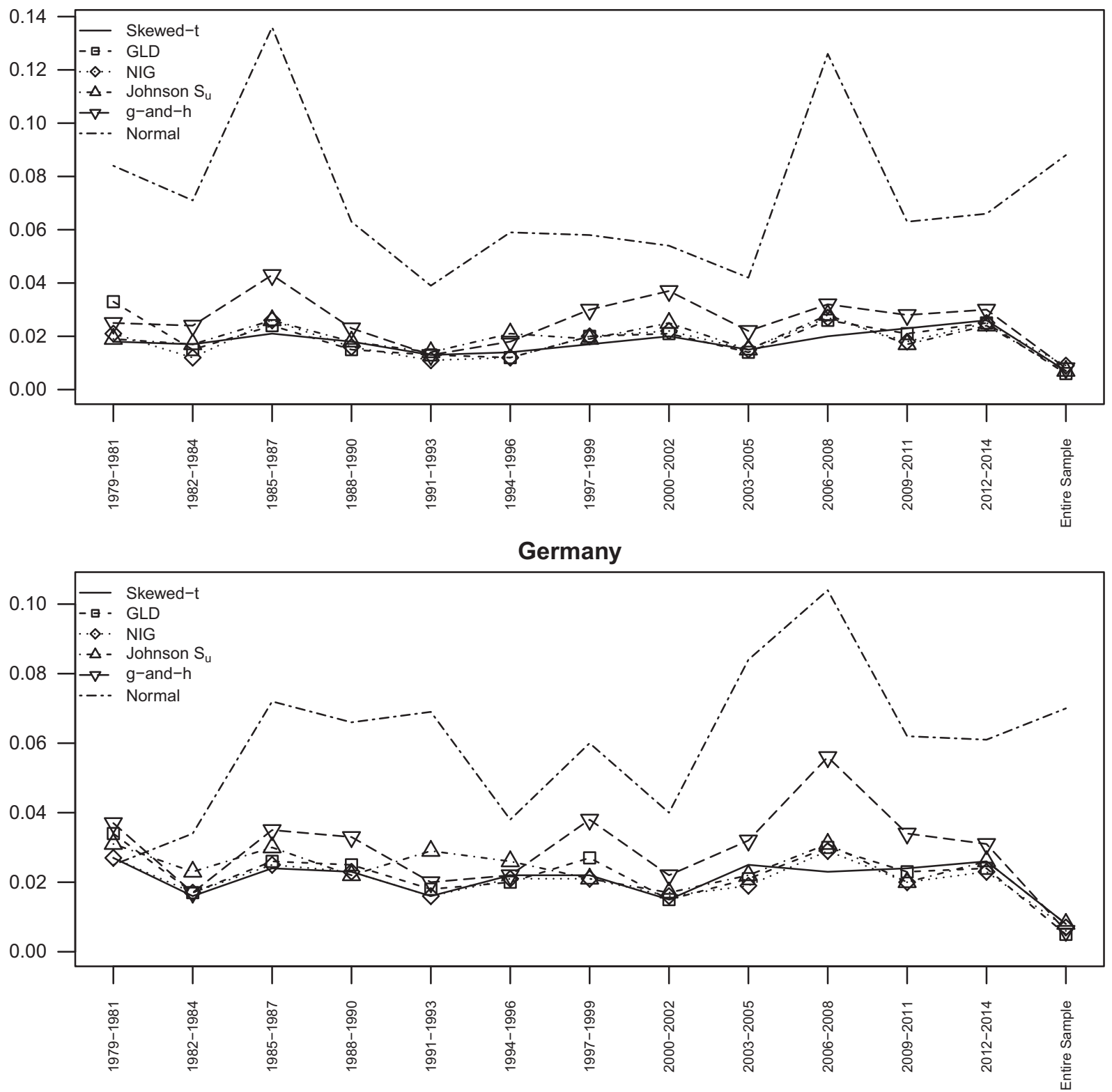

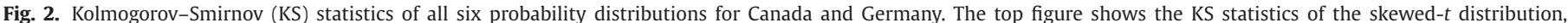

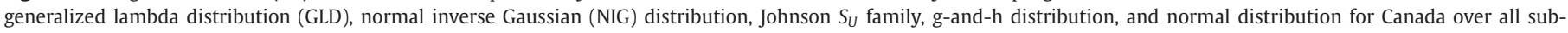

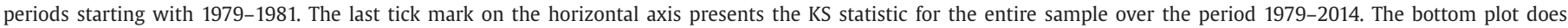
the same for Germany.

distribution takes the following form:

$X_{g, h}(Z)=A+B\left(e^{g Z}-1\right) \frac{\exp \left(h Z^{2} / 2\right)}{g}$.

Among the alternative fitting methodologies that are proposed for estimating the parameters of the g-and-h distribution, we follow the procedure used in Mills (1995) and Dutta and Babbel (2002) as a result of its tractability. Specifically, the estimation procedure starts with identifying the $p$ th percentile of the parameter $g$ as follows:

$g_{p}=-\left(\frac{1}{Z_{p}}\right) \ln \left(\frac{X_{1-p}-X_{0.5}}{X_{0.5}-X_{p}}\right)$,

where $X_{p}$ and $Z_{p}$ are the $p$ th percentile of the empirical distribution and the standard normal distribution, respectively. It is important to note that by using different values of $p$, one can obtain multiple estimates of g. Following (Mills, 1995), we use nine percentiles and we choose percentiles using letter values; i.e., $p=1 / 2,1 / 4,1 / 16, \ldots$. The estimate for $g$ is calculated as the median of $g_{p}$ values.

Using equation (3.2), it is trivial to derive

$X_{p}=A+B\left(e^{g Z_{p}}-1\right) \frac{\exp \left(h Z_{p}^{2} / 2\right)}{g}$ and

$X_{1-p}=A+B\left(e^{g Z_{(1-p)}}-1\right) \frac{\exp \left(h Z_{(1-p)}^{2} / 2\right)}{g}$.

Since $X_{0.5}=A$, the location parameter $A$ is given by the median of the data set. 


\section{Mexico}

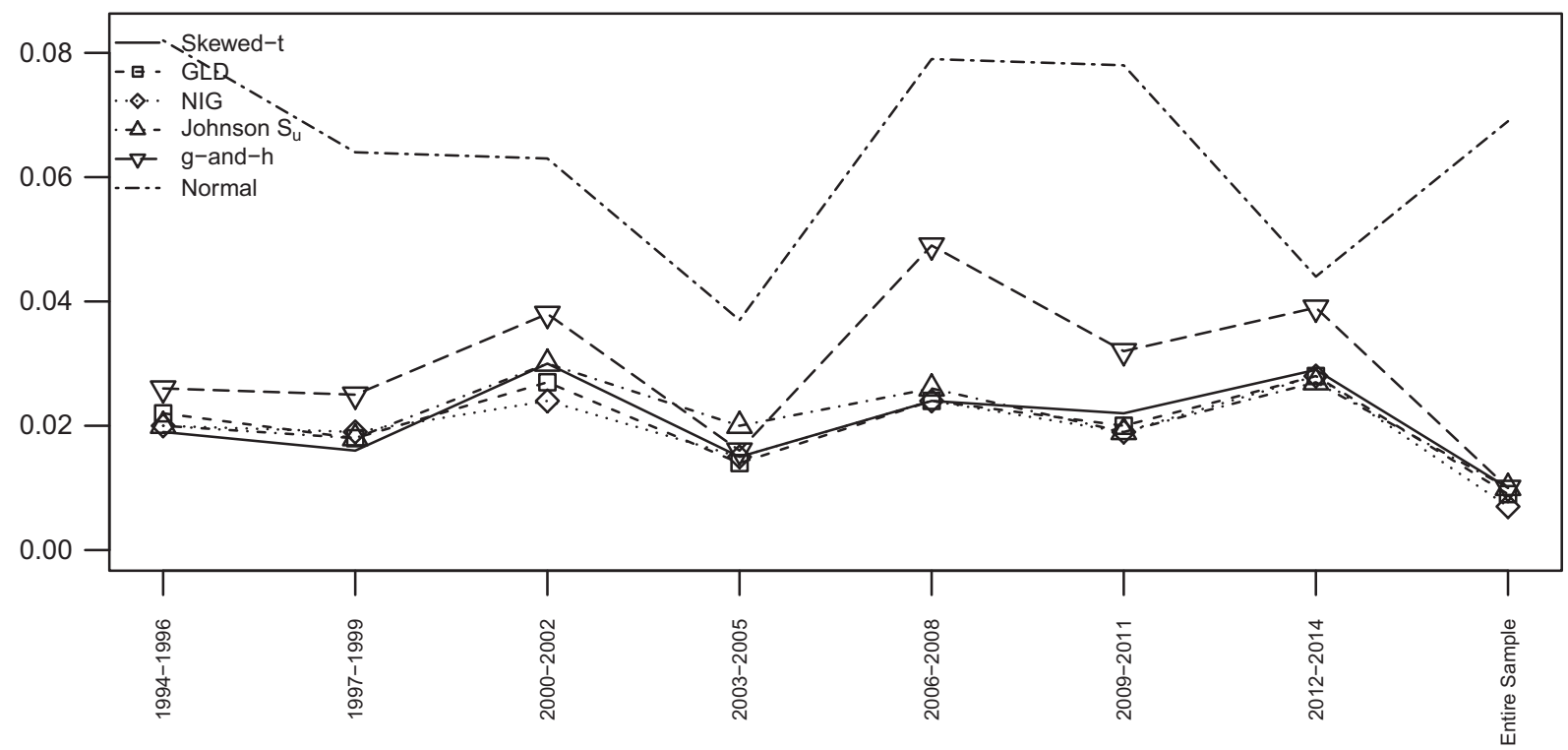

Turkey

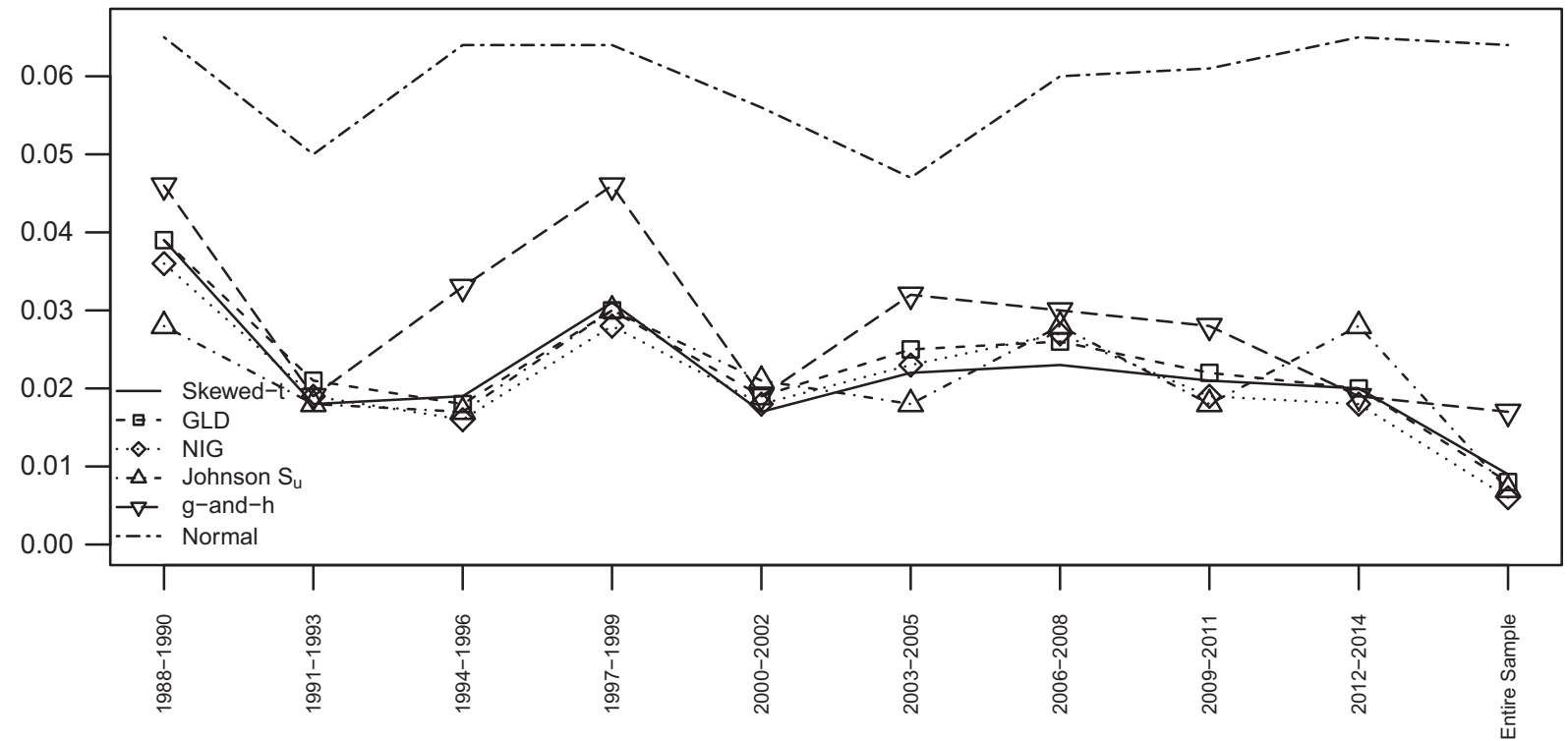

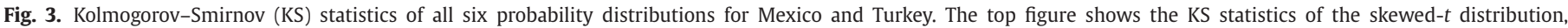

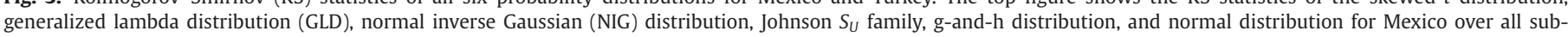

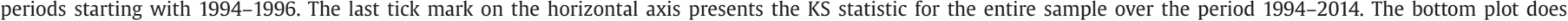
the same for Turkey over all sub-periods starting with 1988-1990.

Subtracting (3.5) from (3.4) and letting $Z_{p}=-Z_{1-p}$ provides the following result:

$\ln \frac{g\left(X_{p}-X_{1-p}\right)}{e^{g Z_{p}}-e^{-g Z_{p}}}=\ln (B)+h\left(Z_{p}^{2} / 2\right)$.

If the data is positively skewed, the left-hand side of (3.6) can be replaced with the upper half-spread (UHS), as defined in Hoaglin (2006):

$\mathrm{UHS}=\frac{g\left(X_{1-p}-X_{0.5}\right)}{\left(e^{-g Z_{p}}-1\right)}$

Conversely, if the data is negatively skewed, then a lower halfspread (LHS) can be used on the left-hand side of (3.6):

LHS $=\frac{g\left(X_{0.5}-X_{p}\right)}{\left(1-e^{g Z_{p}}\right)}$
The estimates of $h$ and $B$ are obtained from the coefficient and the intercept of the linear regression of $\operatorname{In}(\mathrm{UHS})$ and $\operatorname{In}(\mathrm{LHS})$ on $\left(Z_{p}^{2} / 2\right)$.

\section{Performance and risk estimation}

The goal of this section is to evaluate the performances of the density functions described in Section 3 in order to model the daily equity index returns. To this end, we use the Kolmogorov-Smirnov (KS) and Anderson-Darling (AD) test statistics in Section 4.1 and we present the Value-at-Risk (VaR) levels in Section 4.2 .

\subsection{Goodness-of-fit}

In this section, we first use the $\mathrm{KS}$ and $\mathrm{AD}$ test statistics to compare the goodness-of-fit of the distributions of interest. Both 


\section{Canada}

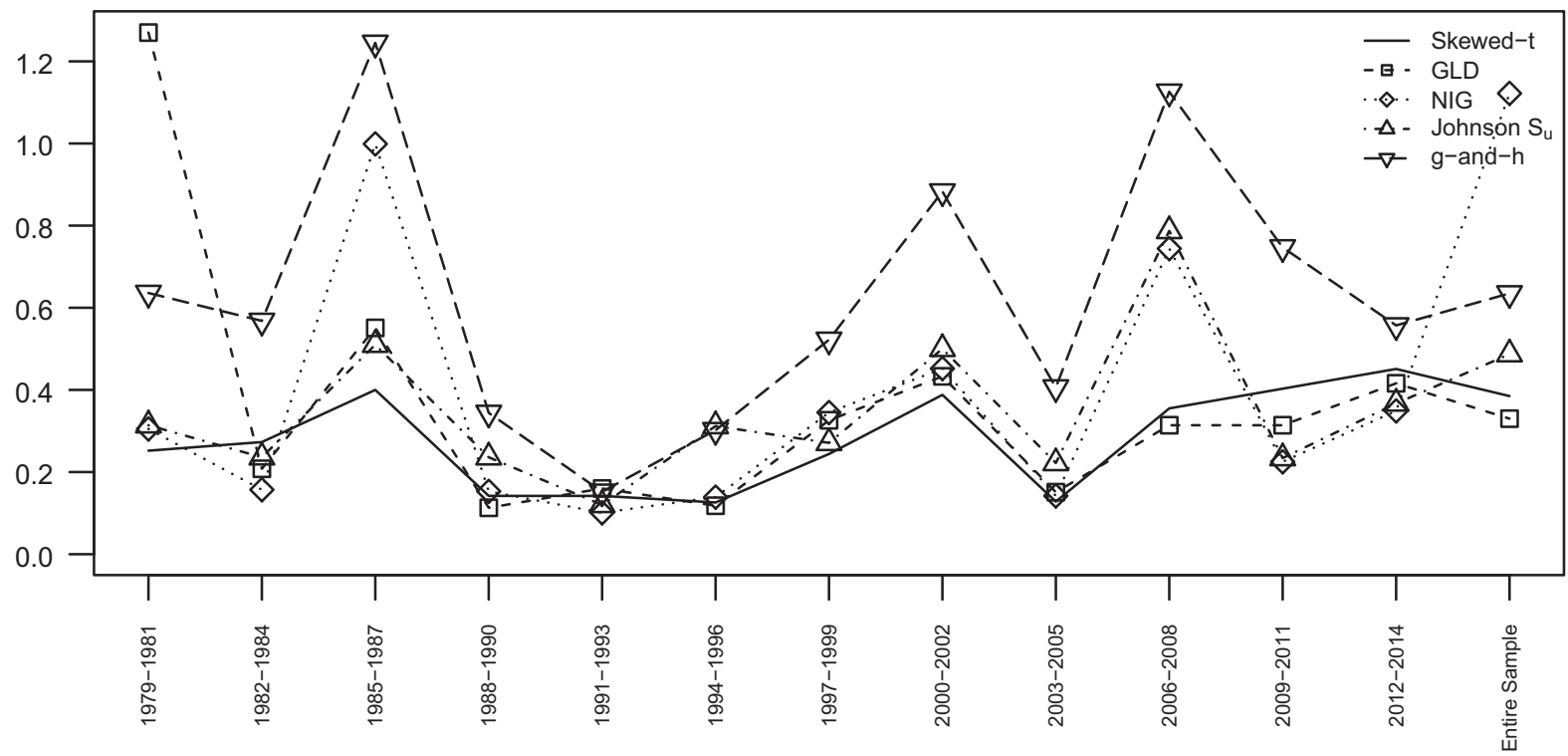

Germany

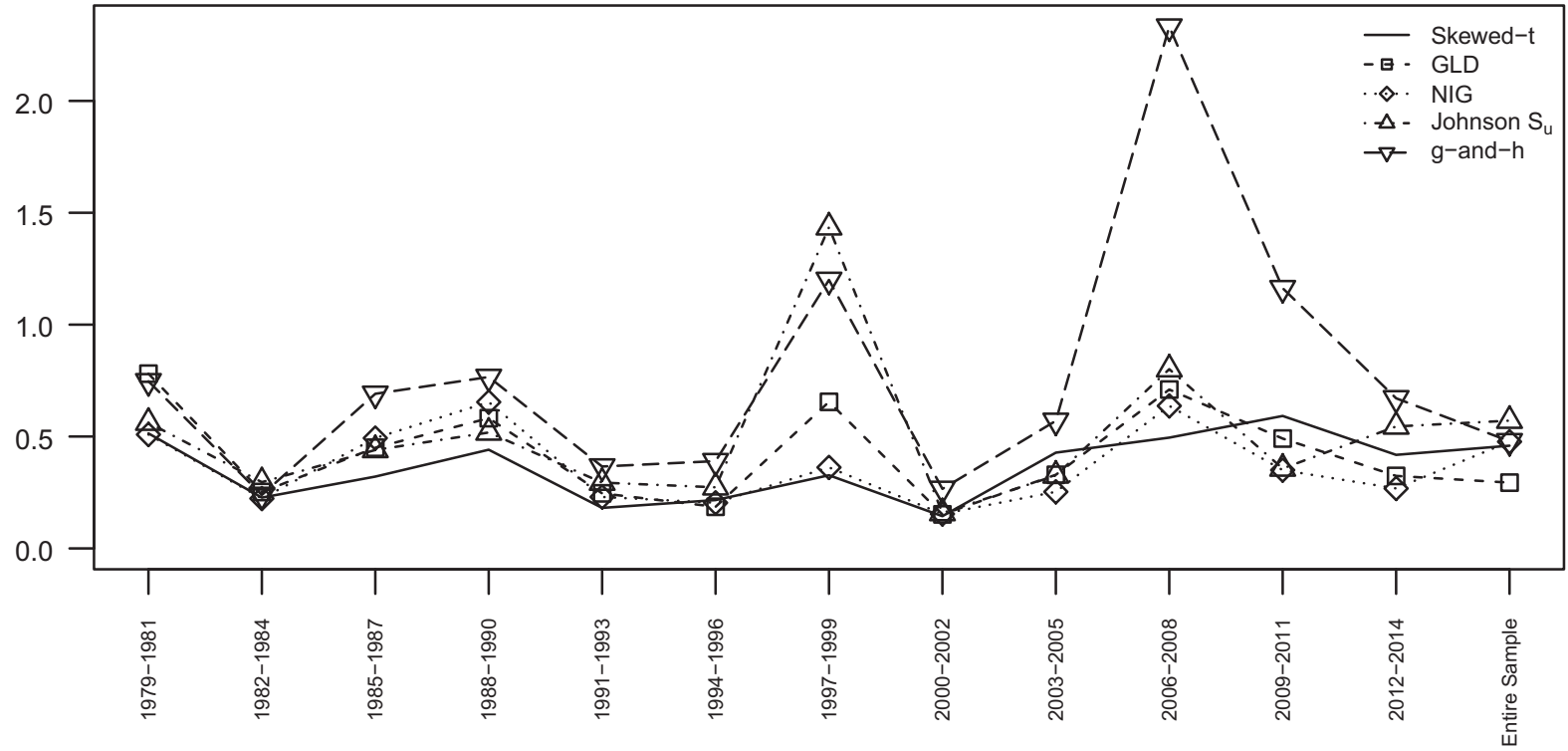

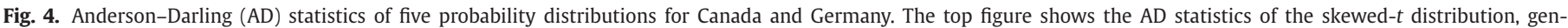

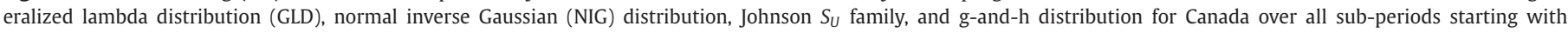

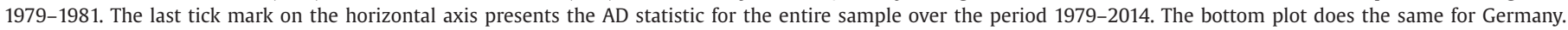

of these test statistics summarize the difference between the fitted cumulative distribution function $\hat{F}$ and the empirical cumulative distribution function $F_{e}$. In particular, the KS test statistic corresponds to the largest distance between $F_{e}(x)$ and $\hat{F}(x)$, that is, $\sup _{x}\left\{\left|F_{e}(x)-\hat{F}(x)\right|\right\}$, while the AD test statistic corresponds to the weighted average of the squared differences, $\left(F_{e}(x)-\hat{F}(x)\right)^{2}$, where the weights are chosen in such a way that the discrepancies in the tails are emphasized. The smaller the KS and AD test statistics, the better the fit.

Fig. 2 presents the computed KS test statistics for the developed markets of Canada and Germany under the six distributional assumptions. Fig. 3 does the same for the emerging markets of Mexico and Turkey. Fig. 4 presents the computed AD test statistics for the developed markets of Canada and Germany using all distributions except the normal distribution. Fig. 5 does the same for the emerging markets of Mexico and Turkey. The markets on the plots have been selected as they are the most representative of the overall results. We excluded the normal distribution from Figs. 4 and 5 since the $A D$ test statistic values for the normal distribution are too large compared to the respective results for all other distributions under consideration, and when the normal distribution's $\mathrm{AD}$ statistics are included, the scale of $\mathrm{y}$-axis becomes very large, which prevents to reveal the differences of $A D$ statistics for other distributions. KS and $\mathrm{AD}$ plots for the remaining markets can be found in the Appendix.

We find that for every market in each sub-sample, and in the entire sample, the KS statistic in the normal case is almost always above the KS statistics in all other five distributions. A similar argument also holds for the AD statistics. Furthermore, we observe that, in general, all five distributions excluding the g-and-h distribution perform very similarly to each other; the g-and-h distribution marginally underperforms in many sub-samples. 


\section{Mexico}

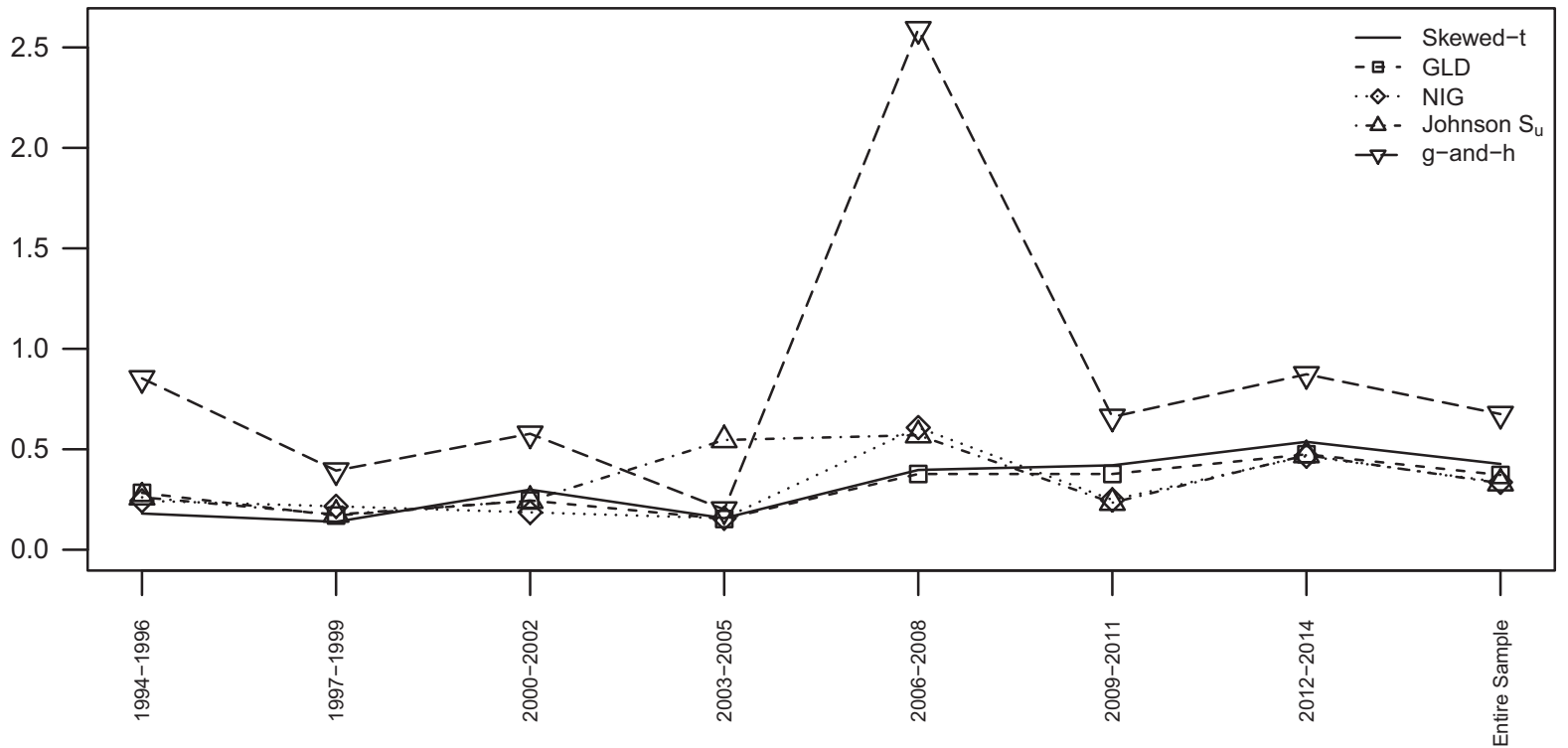

Turkey

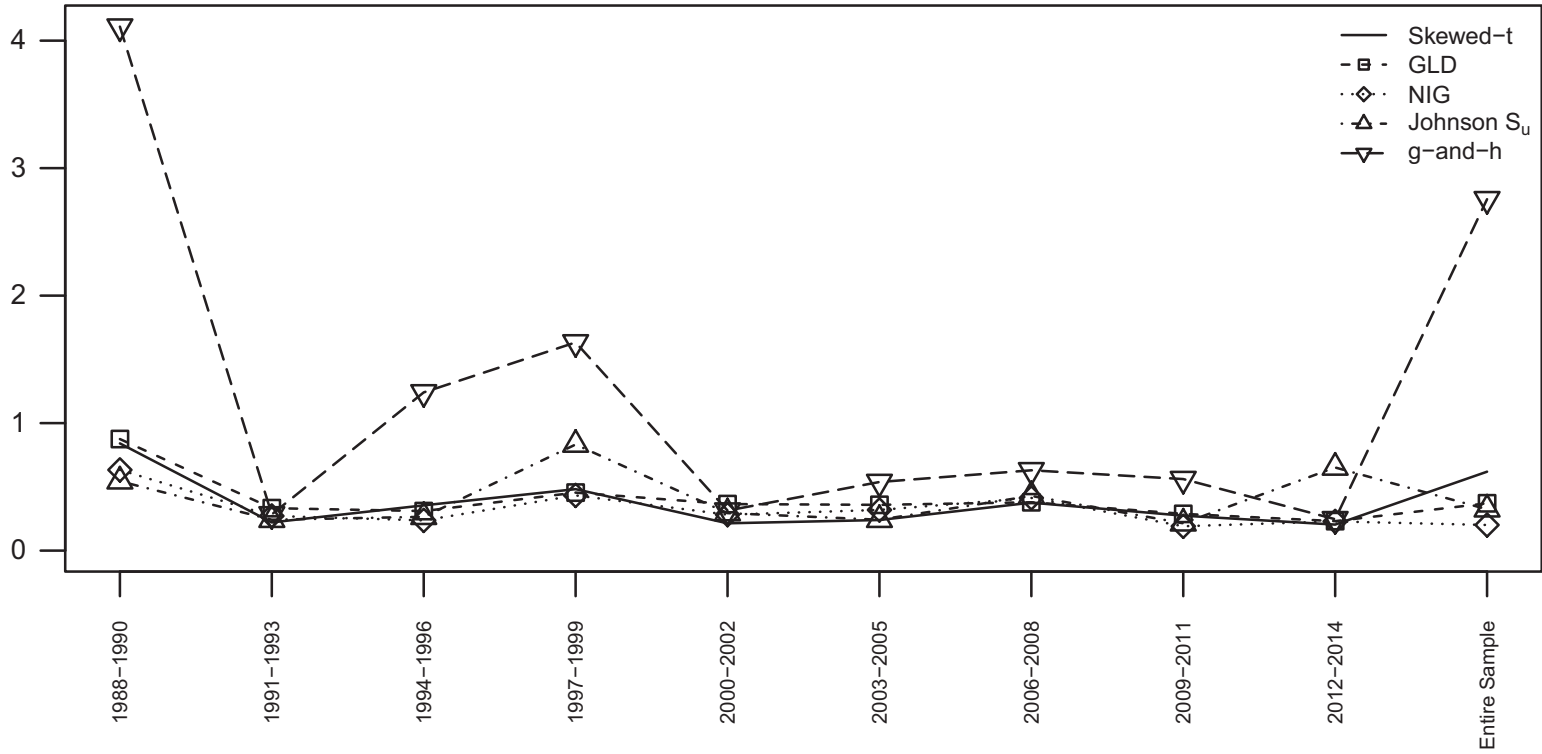

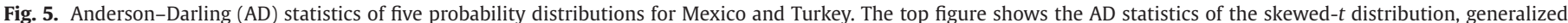

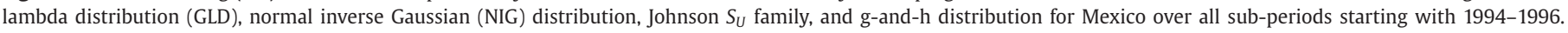

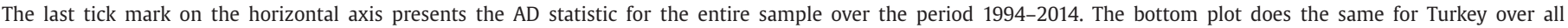
sub-periods starting with 1988-1990.

Next, we compare the distributions of interest by plotting the empirical histogram of the daily returns together with the estimated distribution functions. Fig. 6 presents the empirical histogram of the log-returns of the Ibovespa Index (Brazil) over the sub-period 1997-1999 together with six estimated distribution functions for the same index. One notable observation is that the normal distribution significantly underperforms other distributions in modeling the Ibovespa Index. In particular, the normal distribution cannot capture the peakedness and the tails of the data. Other distributions including the generalized lambda distribution (GLD), the skewed- $t$ distribution, the normal inverse Gaussian (NIG) distribution, and the g-and-h distribution perform very similarly in modeling the data. However, the fit of the Johnson translation system is slightly more peaked than the histogram.

As the computed $\mathrm{KS}$ and $\mathrm{AD}$ statistic values are very close to each other in all distributions, it is difficult to identify whether the differences in these statistics are significant. ${ }^{8}$ Furthermore, Fig. 6 does not provide an answer to the question of which distribution models the daily equity return data best. To address this problem, we conduct a power test, where we calculate the $p$-value of each fit. The null hypothesis is that the observed data originates from the hypothesized distribution and the alternative hypothesis is that the observed data does not belong to the hypothesized distribution. The null hypothesis is rejected if the $p$-value is lower than $\alpha=5 \%$. It is important to recognize here that for many distributions under consideration, the critical values of both the KS statistic and AD statistic do not exist. The typical approach in this case is to compute the $p$-values and the critical values by means of a

${ }^{8}$ We must express our gratitude to an anonymous referee for his/her observation. 
Brazil (1997-1999)

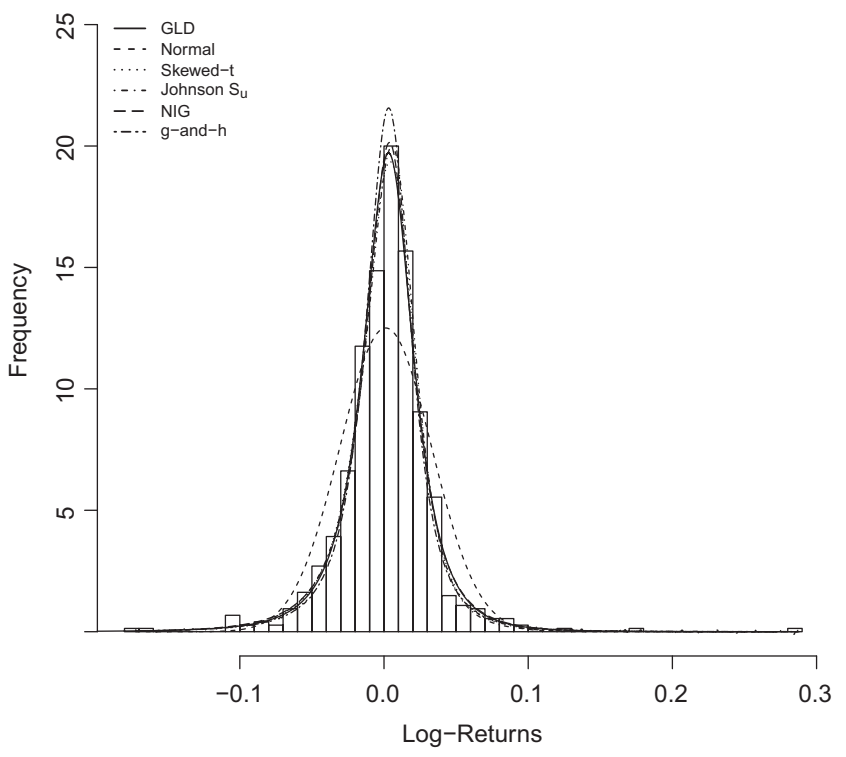

Fig. 6. Histogram and distribution fits for a selected market. This figure plots the histogram of the Brazil log-returns together with the estimated distribution functions of the generalized lambda distribution (GLD), normal distribution, skewed- $t$ distribution, Johnson $S_{U}$ family, normal inverse Gaussian (NIG) distribution, and gand-h distribution over the sub-period 1997-1999. The bins of the histogram are calculated with the Scott method in R.

Monte Carlo simulation for each hypothesized distribution (Ross, 2004). We follow the following steps to calculate the $p$-value:

Step 1. Use the data to estimate the parameters of the hypothesized distribution and compute the value of the test statistics denoted by $G$ (such as KS and/or AD statistic).

Step 2. Generate a sample of size $n$ that is equal to the number of observed data from the fitted distribution.

Step 3. Fit the hypothesized distribution to the data generated in Step 2 and estimate the sample goodness-of-fit statistics.

Step 4: Repeat Step 1 through Step 31000 times and calculate the $p$-value as the proportion of times the sample statistics values exceed the observed value $G$ of the original sample.

Step 5: Reject the null hypothesis if $p$-value is smaller than 0.05 .

Table 3 tabulates the number of rejections using both KS and $\mathrm{AD}$ test statistics in each sub-period for each distribution in the developed markets. Table 4 does the same for emerging markets. ${ }^{9}$ The last row of both tables tabulates the percentage of sub-periods (out of 12 sub-periods) in which a particular distribution is accepted by the $p$-value test using both KS and AD statistics. The results reported in both tables suggest that the GLD is a prominent alternative for fitting the daily equity index return data. We fail to reject the GLD according to both KS and AD test statistics for all index returns in all sub-samples, as well as in the entire sample. Therefore, according to the $p$-value test, we can conclude that the GLD is a powerful distribution in modeling both the center and the tails of the daily index return data.

We conclude this section by comparing the models considered in this paper according to their stability. ${ }^{10}$ Stability is particularly important for applications of portfolio analysis and risk management (Rachev \& Mittnik, 2000). In particular, stable distributed

\footnotetext{
${ }^{9}$ To save space, we do not report the results for each specific market here. However, these results are available upon request.

${ }^{10}$ We must express our gratitude to an anonymous referee who brought this to our attention.
}

returns possess the property that linear combinations of return series, such as portfolios, follow a stable distribution. We investigate the relative stability of the models of interest by comparing their capability to represent the variety of shapes taken by return distributions over twelve sub-periods. We find that in both emerging and developed markets, GLD can adequately describe the empirical distributions in all sub-periods. Other distributions achieve a good fit in smaller share of periods. Specifically, in developed markets, GLD is followed by Johnson $S_{U}$ family (92\%), g-and-h distribution (67\%), skewed- $t$ distribution (42\%), NIG distribution (41\%), and normal distribution (0\%) under the KS statistic and by g-and-h distribution (67\%), Johnson $S_{U}$ family (42\%), skewed-t distribution (25\%), NIG distribution (17\%), and normal distribution (0\%) under the AD statistic (the last row of Table 3). In emerging markets, both Johnson $S_{U}$ family and g-and-h distribution follow GLD with a share of $67 \%$, and skewed- $t$ distribution and NIG distribution with a share of $25 \%$ under the KS statistic. When the AD statistic is used to perform the $p$-value test, the GLD is accepted in all of the sub-periods, the Johnson $S_{U}$ family and g-and-h are accepted in $58 \%$ of periods, and the skewed- $t$ distribution and NIG distribution are accepted in $25 \%$ and $17 \%$ of periods, respectively. The normal distribution is not accepted in any of the sub-periods (the last row of Table 4).

\subsection{Risk estimation}

We examine the behavior of the distributions at the extreme values of each market index return using the VaR measure. The purpose of this investigation is to observe the risk that an investor is facing when she has a long or short position on the market indexes from our sample. The risk levels are determined as $\alpha \in$ $\{0.005,0.01,0.05,0.95,0.99,0.995\}$, in which the first three levels represent the lower extreme of returns (long position) and the last three represent the upper extreme of returns (short position). Insample $\operatorname{VaR}(\alpha)$ values are calculated to observe the behavior of the distributions at the tails. We then apply the Kupiec likelihood ratio test given in Kupiec (1995), which tests whether the expected proportion of violations is equal to $\alpha$. The likelihood ratio test statistic is given by:

$2 \log \left((\tau(\alpha) / n)^{\tau(\alpha)}(1-\tau(\alpha) / n)^{n-\tau(\alpha)}\right)-2 \log \left(\alpha^{\tau(\alpha)}(1-\alpha)^{n-\tau(\alpha)}\right)$,

where $\tau(\alpha)$ is the number of times the observed returns are above (short positions) or below (long positions) the theoretical VaR value and $n$ is the sample size.

The results of the Kupiec test are represented in Tables 5 and 6. For example, Table 5 lists the number of times each distribution is rejected at each significance level for all of the developed markets in each sub-period. Table 6 does the same for emerging markets. Focusing on the sub-period 1979-1981 in Table 5, we see that the GLD, Johnson $S_{U}$ family, skewed- $t$ distribution, NIG distribution, and g-and-h are not rejected in any of the significance levels. However, the normal distribution is rejected by 3 markets at the significance level of 0.005 , by 2 markets at the significance level of 0.01 , and by 1 market at the significance level of 0.95 . The total number of rejections is tabulated at the far right column of the table. For the normal distribution, for instance, the total number of rejections is equal to 6 over the sub-period 1979-1981. Adding up the number of rejections over the sub-periods, we see that the GLD is the least rejected model with only 7 rejections in the developed markets and 8 rejections in the emerging markets. On the other hand, the normal distribution is the most rejected model with 207 rejections in the developed markets and 213 rejections in the emerging markets. In both developed and emerging markets, the g-and-h distribution, NIG distribution, and skewed$t$ distribution perform similarly to each other, while the Johnson $S_{U}$ family underperforms other distributions with 65 rejections in the developed markets and 57 rejections in the emerging markets. 
Table 3

Number of times that each distribution is rejected according to Kolmogorov-Smirnov (KS) and AndersonDarling (AD) test statistics in developed markets. The first column of this table (excluding the last row) lists the periods under consideration; the second column (excluding the last row) presents the number of developed markets considered in each period; the remaining columns (excluding the last row) presents the number of times a distribution is rejected by the p-value test according to the KS and AD test statistics. For instance, in the sub-period 1979-1981, the generalized lambda distribution (GLD), Johnson $S_{U}$ family, and g-and-h distribution are rejected neither according to the KS statistic nor according to the AD statistic; the skewed- $t$ distribution is rejected once according to both $\mathrm{KS}$ and $\mathrm{AD}$ test statistics; the normal inverse Gaussian (NIG) distribution is not rejected according to the KS statistic but rejected once according to the AD statistic; and the normal distribution is rejected 3 times according to both KS and AD statistics. The very last row of this table presents the percentage of periods in which a particular distribution is accepted by the $p$-value test. For instance, the GLD is never rejected in any of the sub-periods; therefore, its percentage of acceptance is $100 \%$ according to both KS and AD statistics. The Johnson $S_{U}$ family is rejected in the sub-period 2003-2005 according to the KS statistic (rejected only once in 12 sub-periods; thus, the percentage of acceptance is $11 / 12 \times 100 \approx 92 \%$ ) and is not rejected in the sub-periods 1979-1981, 1988-1990, 1994-1996, 2009-2011, and 2012-2014 according to the AD statistic (accepted in 5 sub-periods out of 12 sub-periods; thus, the percentage of acceptance is $5 / 12 \times 100 \approx$ $42 \%)$.

\begin{tabular}{|c|c|c|c|c|c|c|c|c|c|c|c|c|c|}
\hline \multirow[t]{2}{*}{ Period } & \multirow[t]{2}{*}{ \# Markets } & \multicolumn{2}{|l|}{ GLD } & \multicolumn{2}{|c|}{ Johnson $S_{U}$} & \multicolumn{2}{|c|}{ Skewed-t } & \multicolumn{2}{|c|}{ NIG } & \multicolumn{2}{|c|}{ g-and-h } & \multicolumn{2}{|c|}{ Normal } \\
\hline & & KS & $\mathrm{AD}$ & KS & $A D$ & KS & $A D$ & KS & $A D$ & KS & $A D$ & KS & $A D$ \\
\hline 1979-1981 & 4 & 0 & 0 & 0 & 0 & 1 & 1 & 0 & 1 & 0 & 0 & 3 & 3 \\
\hline 1982-1984 & 5 & 0 & 0 & 0 & 1 & 0 & 0 & 0 & 0 & 0 & 0 & 4 & 4 \\
\hline 1985-1987 & 7 & 0 & 0 & 0 & 1 & 1 & 2 & 3 & 4 & 0 & 0 & 7 & 7 \\
\hline 1988-1990 & 8 & 0 & 0 & 0 & 0 & 0 & 2 & 1 & 4 & 0 & 0 & 8 & 8 \\
\hline 1991-1993 & 9 & 0 & 0 & 0 & 1 & 2 & 3 & 1 & 3 & 1 & 0 & 8 & 8 \\
\hline 1994-1996 & 9 & 0 & 0 & 0 & 0 & 1 & 1 & 0 & 1 & 0 & 0 & 8 & 8 \\
\hline 1997-1999 & 10 & 0 & 0 & 0 & 1 & 0 & 0 & 2 & 0 & 0 & 0 & 8 & 9 \\
\hline 2000-2002 & 10 & 0 & 0 & 0 & 1 & 0 & 0 & 0 & 1 & 0 & 0 & 9 & 10 \\
\hline 2003-2005 & 10 & 0 & 0 & 2 & 7 & 2 & 3 & 2 & 2 & 1 & 1 & 10 & 10 \\
\hline 2006-2008 & 10 & 0 & 0 & 0 & 3 & 0 & 2 & 3 & 4 & 2 & 1 & 10 & 10 \\
\hline 2009-2011 & 10 & 0 & 0 & 0 & 0 & 1 & 2 & 0 & 1 & 2 & 2 & 9 & 10 \\
\hline 2012-2014 & 10 & 0 & 0 & 0 & 0 & 1 & 3 & 1 & 1 & 0 & 0 & 10 & 10 \\
\hline Entire Sample & 10 & 0 & 0 & 1 & 2 & 2 & 7 & 2 & 7 & 1 & 1 & 10 & 10 \\
\hline$\%$ of acceptance & & 100 & 100 & 92 & 42 & 42 & 25 & 41 & 17 & 67 & 67 & 0 & 0 \\
\hline
\end{tabular}

Table 4

Number of times that each distribution is rejected according to Kolmogorov-Smirnov (KS) and AndersonDarling (AD) test statistics in emerging markets. The first column of this table (excluding the last row) lists the periods under consideration; the second column (excluding the last row) presents the number of emerging markets considered in each period; the remaining columns (excluding the last row) presents the number of times a distribution is rejected by the $p$-value test according to the KS and AD statistics. For instance, in the sub-period 1979-1981, the generalized lambda distribution (GLD), Johnson $S_{U}$ family, normal inverse Gaussian (NIG) distribution, and g-and-h distribution are rejected neither according to the KS statistic nor according to the AD statistic; the skewed-t distribution is rejected once according to the AD statistic and normal distribution is rejected 2 times according to both $\mathrm{KS}$ and $\mathrm{AD}$ statistics. The very last row of this table presents the percentage of periods in which a particular distribution is accepted by the $p$-value test. For instance, the GLD is never rejected in any of the sub-periods; therefore, its percentage of acceptance is $100 \%$ according to both KS and AD statistics. The Johnson $S_{U}$ family is rejected in the sub-periods 1982-1984, 1985-1987, 1988-1990, and 2009-2011 according to the KS statistic (rejected 4 times in 12 sub-periods; thus, the percentage of acceptance is $8 / 12 \times 100 \approx 67 \%$ ) and is rejected in the sub-periods 1985-1987, 1991-1993, 1997-1999, 2003-2005, and 2009-2011 according to the AD statistic (rejected in 5 sub-periods out of 12 sub-periods; thus, the percentage of acceptance is $7 / 12 \times 100 \approx 58 \%$ ).

\begin{tabular}{|c|c|c|c|c|c|c|c|c|c|c|c|c|c|}
\hline \multirow[t]{2}{*}{ Period } & \multirow[t]{2}{*}{ \# Markets } & \multicolumn{2}{|l|}{ GLD } & \multicolumn{2}{|c|}{ Johnson $S_{U}$} & \multicolumn{2}{|c|}{ Skewed-t } & \multicolumn{2}{|c|}{ NIG } & \multicolumn{2}{|c|}{ g-and-h } & \multicolumn{2}{|c|}{ Normal } \\
\hline & & KS & $A D$ & KS & $\mathrm{AD}$ & KS & $A D$ & KS & $A D$ & KS & $A D$ & KS & $\mathrm{AD}$ \\
\hline 1979-1981 & 2 & 0 & 0 & 0 & 0 & 0 & 1 & 0 & 0 & 0 & 0 & 2 & 2 \\
\hline 1982-1984 & 2 & 0 & 0 & 1 & 0 & 0 & 0 & 1 & 1 & 0 & 0 & 2 & 2 \\
\hline 1985-1987 & 2 & 0 & 0 & 1 & 2 & 2 & 2 & 2 & 2 & 0 & 0 & 2 & 2 \\
\hline 1988-1990 & 4 & 0 & 0 & 1 & 0 & 2 & 2 & 2 & 1 & 0 & 1 & 4 & 4 \\
\hline 1991-1993 & 5 & 0 & 0 & 0 & 1 & 0 & 0 & 0 & 0 & 1 & 1 & 4 & 4 \\
\hline 1994-1996 & 7 & 0 & 0 & 0 & 0 & 2 & 1 & 1 & 1 & 0 & 0 & 6 & 6 \\
\hline 1997-1999 & 9 & 0 & 0 & 0 & 2 & 3 & 2 & 2 & 2 & 1 & 1 & 8 & 9 \\
\hline 2000-2002 & 9 & 0 & 0 & 0 & 0 & 1 & 0 & 0 & 2 & 0 & 0 & 8 & 8 \\
\hline 2003-2005 & 10 & 0 & 0 & 0 & 2 & 2 & 2 & 1 & 2 & 1 & 1 & 7 & 8 \\
\hline 2006-2008 & 10 & 0 & 0 & 0 & 0 & 2 & 2 & 1 & 2 & 2 & 2 & 10 & 10 \\
\hline 2009-2011 & 10 & 0 & 0 & 1 & 1 & 1 & 1 & 1 & 1 & 0 & 0 & 10 & 10 \\
\hline 2012-2014 & 10 & 0 & 0 & 0 & 0 & 1 & 1 & 2 & 2 & 0 & 0 & 9 & 9 \\
\hline Entire Sample & 10 & 0 & 0 & 1 & 2 & 4 & 5 & 4 & 5 & 4 & 4 & 10 & 10 \\
\hline$\%$ of acceptance & & 100 & 100 & 67 & 58 & 25 & 25 & 25 & 17 & 67 & 58 & 0 & 0 \\
\hline
\end{tabular}


Table 5

Value-at-Risk (VaR) failure rate results for the developed markets. This table presents the number of times each distribution including the generalized lambda distribution (GLD), Johnson $S_{U}$ family, skewed- $t$ distribution, normal inverse Gaussian (NIG) distribution, g-and-h distribution, and normal distribution is rejected at various significance levels for all developed markets in each sub-period according to the Kupiec likelihood ratio test.

\begin{tabular}{|c|c|c|c|c|c|c|c|c|c|}
\hline \multirow[t]{2}{*}{ Period } & \multirow[t]{2}{*}{ \# Market } & \multirow[t]{2}{*}{ Method } & \multicolumn{6}{|c|}{ Significance levels } & Total \# Rejections \\
\hline & & & 0.005 & 0.01 & 0.05 & 0.95 & 0.99 & 0.995 & \\
\hline 1979-1981 & 4 & GLD & 0 & 0 & 0 & 0 & 0 & 0 & 0 \\
\hline & & Johnson $S_{U}$ & 0 & 0 & 0 & 0 & 0 & 0 & 0 \\
\hline & & Skewed-t & 0 & 0 & 0 & 0 & 0 & 0 & 0 \\
\hline & & NIG & 0 & 0 & 0 & 0 & 0 & 0 & 0 \\
\hline & & g-and-h & 0 & 0 & 0 & 0 & 0 & 0 & 0 \\
\hline & & Normal & 3 & 2 & 0 & 1 & 0 & 0 & 6 \\
\hline 1982-1984 & 5 & GLD & 0 & 0 & 0 & 0 & 0 & 0 & 0 \\
\hline & & Johnson $S_{U}$ & 1 & 1 & 0 & 0 & 1 & 2 & 5 \\
\hline & & Skewed-t & 0 & 0 & 0 & 0 & 0 & 0 & 0 \\
\hline & & NIG & 0 & 0 & 0 & 0 & 0 & 0 & 0 \\
\hline & & g-and-h & 0 & 0 & 0 & 0 & 0 & 0 & 0 \\
\hline & & Normal & 1 & 0 & 0 & 0 & 2 & 3 & 6 \\
\hline 1985-1987 & 7 & GLD & 0 & 0 & 0 & 0 & 0 & 0 & 0 \\
\hline & & Johnson $S_{U}$ & 1 & 0 & 0 & 0 & 0 & 0 & 1 \\
\hline & & Skewed-t & 0 & 0 & 0 & 0 & 0 & 0 & 0 \\
\hline & & NIG & 0 & 0 & 1 & 0 & 0 & 0 & 1 \\
\hline & & g-and-h & 1 & 1 & 0 & 0 & 0 & 0 & 2 \\
\hline & & Normal & 1 & 1 & 3 & 4 & 0 & 0 & 9 \\
\hline 1988-1990 & 8 & GLD & 0 & 0 & 0 & 1 & 0 & 0 & 1 \\
\hline & & Johnson $S_{U}$ & 1 & 2 & 0 & 0 & 1 & 1 & 5 \\
\hline & & Skewed-t & 0 & 0 & 0 & 0 & 0 & 0 & 0 \\
\hline & & NIG & 0 & 0 & 0 & 0 & 0 & 0 & 0 \\
\hline & & g-and-h & 0 & 0 & 0 & 0 & 0 & 0 & 0 \\
\hline & & Normal & 4 & 4 & 2 & 5 & 0 & 1 & 16 \\
\hline 1991-1993 & 9 & GLD & 0 & 0 & 0 & 0 & 0 & 0 & 0 \\
\hline & & Johnson $S_{U}$ & 2 & 2 & 0 & 0 & 1 & 1 & 6 \\
\hline & & Skewed-t & 0 & 0 & 0 & 0 & 0 & 0 & 0 \\
\hline & & NIG & 0 & 0 & 0 & 1 & 0 & 0 & 1 \\
\hline & & g-and-h & 0 & 0 & 0 & 0 & 0 & 0 & 0 \\
\hline & & Normal & 1 & 0 & 2 & 1 & 0 & 4 & 8 \\
\hline 1994-1996 & 9 & GLD & 0 & 0 & 0 & 0 & 0 & 0 & 0 \\
\hline & & Johnson $S_{U}$ & 1 & 0 & 0 & 0 & 1 & 0 & 2 \\
\hline & & Skewed-t & 0 & 0 & 0 & 0 & 0 & 0 & 0 \\
\hline & & NIG & 0 & 0 & 0 & 0 & 0 & 0 & 0 \\
\hline & & g-and-h & 0 & 0 & 0 & 0 & 0 & 0 & 0 \\
\hline & & Normal & 5 & 3 & 0 & 0 & 0 & 1 & 9 \\
\hline 1997-1999 & 10 & GLD & 0 & 0 & 0 & 0 & 0 & 0 & 0 \\
\hline & & Johnson $S_{U}$ & 2 & 0 & 0 & 0 & 1 & 1 & 4 \\
\hline & & Skewed-t & 0 & 0 & 0 & 0 & 0 & 0 & 0 \\
\hline & & NIG & 0 & 0 & 0 & 0 & 0 & 0 & 0 \\
\hline & & g-and-h & 0 & 0 & 0 & 0 & 0 & 0 & 0 \\
\hline & & Normal & 5 & 4 & 0 & 3 & 0 & 0 & 12 \\
\hline $2000-2002$ & 10 & GLD & 0 & 0 & 0 & 0 & 0 & 0 & 0 \\
\hline & & Johnson $S_{U}$ & 1 & 0 & 0 & 0 & 0 & 1 & 2 \\
\hline & & Skewed-t & 0 & 0 & 0 & 0 & 0 & 1 & 1 \\
\hline & & NIG & 0 & 0 & 0 & 0 & 0 & 0 & 0 \\
\hline & & g-and-h & 0 & 0 & 0 & 0 & 0 & 0 & 0 \\
\hline & & Normal & 2 & 2 & 0 & 0 & 2 & 4 & 10 \\
\hline 2003-2005 & 10 & GLD & 0 & 0 & 0 & 0 & 0 & 0 & 0 \\
\hline & & Johnson $S_{U}$ & 1 & 1 & 0 & 0 & 1 & 3 & 6 \\
\hline & & Skewed-t & 0 & 0 & 0 & 0 & 0 & 0 & 0 \\
\hline & & NIG & 0 & 0 & 0 & 0 & 0 & 0 & 0 \\
\hline & & g-and-h & 0 & 0 & 0 & 0 & 0 & 0 & 0 \\
\hline & & Normal & 9 & 7 & 0 & 2 & 0 & 2 & 20 \\
\hline 2006-2008 & 10 & GLD & 1 & 0 & 0 & 0 & 1 & 0 & 2 \\
\hline & & Johnson $S_{U}$ & 0 & 0 & 0 & 0 & 2 & 3 & 5 \\
\hline & & Skewed-t & 0 & 0 & 0 & 0 & 2 & 0 & 2 \\
\hline & & NIG & 0 & 0 & 0 & 0 & 1 & 0 & 1 \\
\hline & & g-and-h & 0 & 0 & 0 & 0 & 1 & 0 & 1 \\
\hline & & Normal & 10 & 10 & 1 & 8 & 2 & 6 & 37 \\
\hline 2009-2011 & 10 & GLD & 0 & 0 & 0 & 0 & 0 & 0 & 0 \\
\hline & & Johnson $S_{U}$ & 2 & 1 & 0 & 0 & 0 & 2 & 5 \\
\hline & & Skewed-t & 0 & 0 & 0 & 0 & 0 & 0 & 0 \\
\hline & & NIG & 0 & 0 & 0 & 0 & 0 & 0 & 0 \\
\hline & & g-and-h & 1 & 0 & 0 & 0 & 0 & 0 & 1 \\
\hline & & Normal & 8 & 7 & 0 & 0 & 0 & 2 & 17 \\
\hline 2012-2014 & 10 & GLD & 1 & 0 & 0 & 0 & 0 & 0 & 1 \\
\hline & & Johnson $S_{U}$ & 4 & 3 & 0 & 0 & 3 & 2 & 12 \\
\hline & & Skewed-t & 1 & 0 & 0 & 0 & 0 & 0 & 1 \\
\hline & & NIG & 0 & 0 & 0 & 0 & 0 & 0 & 0 \\
\hline & & g-and-h & 0 & 0 & 0 & 0 & 0 & 0 & 0 \\
\hline & & Normal & 7 & 7 & 0 & 0 & 1 & 1 & 16 \\
\hline Entire Sample & 10 & GLD & 0 & 2 & 1 & 0 & 0 & 0 & 3 \\
\hline & & Johnson $S_{U}$ & 3 & 2 & 2 & 2 & 2 & 2 & 13 \\
\hline & & Skewed-t & 1 & 2 & 1 & 0 & 1 & 0 & 5 \\
\hline & & NIG & 1 & 2 & 2 & 0 & 1 & 0 & 6 \\
\hline & & g-and-h & 1 & 0 & 0 & 0 & 0 & 0 & 1 \\
\hline & & Normal & 10 & 10 & 9 & 5 & 7 & 10 & 51 \\
\hline
\end{tabular}


Table 6

Value-at-Risk (VaR) failure rate results for the emerging markets. This table presents the number of times each distribution including the generalized lambda distribution (GLD), Johnson $S_{U}$ family, skewed- $t$ distribution, normal inverse Gaussian (NIG) distribution, g-and-h distribution, and normal distribution is rejected at various significance levels for all emerging markets in each sub-period according to the Kupiec likelihood ratio test.

\begin{tabular}{|c|c|c|c|c|c|c|c|c|c|}
\hline \multirow[t]{2}{*}{ Period } & \multirow[t]{2}{*}{ \# Market } & \multirow[t]{2}{*}{ Method } & \multicolumn{6}{|c|}{ Significance levels } & \multirow[t]{2}{*}{ Total \# Rejections } \\
\hline & & & 0.005 & 0.01 & 0.05 & 0.95 & 0.99 & 0.995 & \\
\hline \multirow[t]{6}{*}{ 1979-1981 } & \multirow[t]{6}{*}{2} & GLD & 0 & 0 & 0 & 0 & 0 & 0 & 0 \\
\hline & & Johnson $S_{U}$ & 0 & 0 & 0 & 0 & 0 & 1 & 1 \\
\hline & & Skewed-t & 0 & 0 & 0 & 0 & 0 & 0 & 0 \\
\hline & & NIG & 0 & 0 & 0 & 0 & 0 & 0 & 0 \\
\hline & & g-and-h & 0 & 0 & 0 & 0 & 0 & 0 & 0 \\
\hline & & Normal & 1 & 1 & 0 & 1 & 0 & 1 & 4 \\
\hline 1982-1984 & 2 & GLD & 0 & 0 & 0 & 0 & 0 & 0 & 0 \\
\hline & & Johnson $S_{U}$ & 0 & 0 & 0 & 0 & 0 & 0 & 0 \\
\hline & & Skewed-t & 0 & 0 & 0 & 0 & 0 & 0 & 0 \\
\hline & & NIG & 0 & 0 & 0 & 0 & 0 & 0 & 0 \\
\hline & & g-and-h & 0 & 0 & 0 & 0 & 0 & 0 & 0 \\
\hline & & Normal & 1 & 0 & 1 & 0 & 2 & 2 & 6 \\
\hline 1985-1987 & 2 & GLD & 0 & 0 & 0 & 0 & 0 & 0 & 0 \\
\hline & & Johnson $S_{U}$ & 1 & 0 & 0 & 0 & 0 & 0 & 1 \\
\hline & & Skewed-t & 0 & 0 & 0 & 0 & 0 & 0 & 0 \\
\hline & & NIG & 0 & 0 & 0 & 0 & 0 & 0 & 0 \\
\hline & & g-and-h & 0 & 0 & 0 & 0 & 0 & 0 & 0 \\
\hline & & Normal & 2 & 1 & 1 & 1 & 0 & 0 & 5 \\
\hline 1988-1990 & 4 & GLD & 0 & 0 & 0 & 0 & 0 & 1 & 1 \\
\hline & & Johnson $S_{U}$ & 2 & 1 & 0 & 0 & 1 & 2 & 6 \\
\hline & & Skewed-t & 0 & 0 & 0 & 0 & 1 & 2 & 3 \\
\hline & & NIG & 0 & 0 & 0 & 0 & 1 & 2 & 3 \\
\hline & & g-and-h & 0 & 0 & 0 & 0 & 0 & 0 & 0 \\
\hline & & Normal & 3 & 2 & 1 & 1 & 2 & 2 & 11 \\
\hline 1991-1993 & 5 & GLD & 0 & 0 & 0 & 0 & 0 & 2 & 2 \\
\hline & & Johnson $S_{U}$ & 0 & 0 & 0 & 0 & 1 & 2 & 3 \\
\hline & & Skewed-t & 0 & 0 & 0 & 0 & 0 & 2 & 2 \\
\hline & & NIG & 0 & 0 & 0 & 0 & 0 & 2 & 2 \\
\hline & & g-and-h & 0 & 0 & 0 & 0 & 0 & 1 & 1 \\
\hline & & Normal & 1 & 1 & 1 & 0 & 3 & 4 & 10 \\
\hline 1994-1996 & 7 & GLD & 0 & 0 & 0 & 0 & 0 & 0 & 0 \\
\hline & & Johnson $S_{U}$ & 2 & 2 & 0 & 0 & 3 & 2 & 9 \\
\hline & & Skewed-t & 0 & 0 & 0 & 0 & 0 & 1 & 1 \\
\hline & & NIG & 0 & 0 & 0 & 0 & 0 & 1 & 1 \\
\hline & & g-and-h & 0 & 0 & 0 & 0 & 0 & 0 & 0 \\
\hline & & Normal & 3 & 1 & 0 & 0 & 4 & 4 & 12 \\
\hline 1997-1999 & 9 & GLD & 0 & 0 & 0 & 0 & 0 & 1 & 1 \\
\hline & & Johnson $S_{U}$ & 1 & 1 & 0 & 0 & 3 & 3 & 8 \\
\hline & & Skewed-t & 0 & 0 & 0 & 0 & 0 & 1 & 1 \\
\hline & & NIG & 0 & 0 & 0 & 0 & 0 & 1 & 1 \\
\hline & & g-and-h & 0 & 0 & 0 & 0 & 2 & 0 & 2 \\
\hline & & Normal & 5 & 3 & 2 & 3 & 3 & 5 & 21 \\
\hline 2000-2002 & 9 & GLD & 0 & 0 & 0 & 0 & 0 & 0 & 0 \\
\hline & & Johnson $S_{U}$ & 2 & 2 & 0 & 0 & 0 & 0 & 4 \\
\hline & & Skewed-t & 0 & 0 & 0 & 0 & 0 & 0 & 0 \\
\hline & & NIG & 0 & 0 & 0 & 0 & 0 & 0 & 0 \\
\hline & & g-and-h & 0 & 0 & 0 & 1 & 0 & 0 & 1 \\
\hline & & Normal & 4 & 3 & 2 & 2 & 1 & 5 & 17 \\
\hline 2003-2005 & 10 & GLD & 0 & 0 & 0 & 0 & 0 & 0 & 0 \\
\hline & & Johnson $S_{U}$ & 0 & 1 & 0 & 0 & 1 & 2 & 4 \\
\hline & & Skewed-t & 0 & 0 & 0 & 0 & 0 & 0 & 0 \\
\hline & & NIG & 0 & 0 & 0 & 0 & 0 & 0 & 0 \\
\hline & & g-and-h & 0 & 0 & 0 & 0 & 0 & 0 & 0 \\
\hline & & Normal & 4 & 4 & 1 & 4 & 1 & 2 & 16 \\
\hline 2006-2008 & 10 & GLD & 1 & 1 & 0 & 0 & 0 & 0 & 2 \\
\hline & & Johnson $S_{U}$ & 2 & 2 & 0 & 0 & 1 & 1 & 6 \\
\hline & & Skewed-t & 1 & 1 & 0 & 0 & 1 & 1 & 4 \\
\hline & & NIG & 0 & 0 & 0 & 0 & 0 & 0 & 0 \\
\hline & & g-and-h & 0 & 0 & 0 & 0 & 1 & 1 & 2 \\
\hline & & Normal & 10 & 10 & 1 & 6 & 2 & 5 & 34 \\
\hline 2009-2011 & 10 & GLD & 1 & 0 & 0 & 0 & 0 & 0 & 1 \\
\hline & & Johnson $S_{U}$ & 2 & 2 & 0 & 0 & 0 & 0 & 4 \\
\hline & & Skewed-t & 1 & 0 & 0 & 0 & 0 & 0 & 1 \\
\hline & & NIG & 1 & 0 & 0 & 0 & 0 & 0 & 1 \\
\hline & & g-and-h & 1 & 0 & 0 & 0 & 0 & 0 & 1 \\
\hline & & Normal & 6 & 7 & 0 & 0 & 3 & 3 & 19 \\
\hline 2012-2014 & 10 & GLD & 0 & 1 & 0 & 0 & 0 & 0 & 1 \\
\hline & & Johnson $S_{U}$ & 1 & 2 & 0 & 0 & 2 & 1 & 6 \\
\hline & & Skewed-t & 0 & 1 & 0 & 0 & 0 & 0 & 1 \\
\hline & & NIG & 0 & 1 & 0 & 0 & 0 & 0 & 1 \\
\hline & & g-and-h & 0 & 0 & 0 & 0 & 0 & 0 & 0 \\
\hline & & Normal & 3 & 2 & 0 & 0 & 1 & 3 & 9 \\
\hline
\end{tabular}


Table 6 (continued)

\begin{tabular}{|c|c|c|c|c|c|c|c|c|c|}
\hline \multirow[t]{2}{*}{ Period } & \multirow[t]{2}{*}{ \# Market } & \multirow[t]{2}{*}{ Method } & \multicolumn{6}{|c|}{ Significance levels } & \multirow[t]{2}{*}{ Total \# Rejections } \\
\hline & & & 0.005 & 0.01 & 0.05 & 0.95 & 0.99 & 0.995 & \\
\hline \multirow[t]{6}{*}{ Entire Sample } & \multirow[t]{6}{*}{10} & GLD & 0 & 0 & 0 & 0 & 0 & 0 & 0 \\
\hline & & Johnson $S_{U}$ & 1 & 1 & 0 & 0 & 1 & 2 & 5 \\
\hline & & Skewed-t & 0 & 0 & 0 & 1 & 0 & 2 & 3 \\
\hline & & NIG & 0 & 0 & 0 & 1 & 0 & 0 & 1 \\
\hline & & g-and-h & 0 & 1 & 1 & 1 & 3 & 1 & 7 \\
\hline & & Normal & 10 & 10 & 7 & 4 & 8 & 10 & 49 \\
\hline
\end{tabular}

Thus, we conclude that the GLD also outperforms other distributions in terms of VaR performance.

\section{Conclusion}

An analysis of the empirical distribution of equity returns is important to both academic researchers and financial experts and has many implications in the calculation of risk measures such as Value-at-Risk (VaR) and the pricing of equity options. Despite its popularity in finance applications, it is now well known that the normal distribution fails to capture certain characteristics of equity return data. This has motivated researchers to investigate flexible distributions with the ability to better represent stock return data. Nevertheless, on a practical level, which distribution best suited for modeling equity stock return data has remained an open question.

This paper contributes to the literature in the following two ways: First, we investigate the relative performance of five widely used flexible distributions in finance, specifically, the generalized lambda distribution (GLD), Johnson system of distributions, skewed Student- $t$ distribution, normal inverse Gaussian (NIG) distribution, and g-and-h distribution, for modeling the distribution of daily equity index returns of ten developed and ten emerging markets over the years 1979-2014. We also use the normal distribution as a benchmark in our analysis. Second, we evaluate the implication of our results for the implementation of the well-known risk measure, VaR. Our analyses support the empirical evidence in the previous research that the behavior of equity returns are far from being normally distributed. We further show that the marginal distribution of daily equity index returns can be well described by the GLD. The relative stability of the GLD also makes it more favorable among other distributions. From a practical expert intelligent system perspective, the GLD has the following advantages: (i) The representation of the GLD as an inverse cumulative distribution function makes it easy to quickly generate random variates from the GLD in a Monte Carlo simulation, which is widely used in risk management and the pricing of derivative securities. (ii) The percentile representation of the GLD makes it convenient to estimate the risk measures, such as VaR and expected shortfall.

In fitting the distributions to the observed data, we must choose among several fitting methods from the previous research. In making this choice, we consider the availability of off-the-shelf algorithms that can be easily used by expert and intelligent systems. If such an algorithm is not available, then we implement the easiest method. One potential limitation of our work is that the implemented fitting methods may not be the "best" methods. This limitation could be overcome by performing an intensive study to examine the relative performance of fitting methods for each distribution considered in this paper. One such study that we are aware of concerns the estimation of the parameters of the GLD (Corlu \& Meterelliyoz, 2015).

Our focus in this paper is on the unconditional distribution of equity returns. As stock returns typically exhibit temporal dependence, considering conditional homoskedastic models such ARMA and conditional heteroskedastic models such as ARCH, GARCH, and ARMA-GARCH models, where the residuals in these models follow flexible distributions considered in this paper, could be of interest from an expert and intelligent systems perspective. Another future research avenue is related to the stability of the probability distributions. As mentioned in the paper, stability is important, especially for portfolio analysis and risk management. An interesting problem to consider is how the performance of the GLD would compare with some of the popular stable distributions used in finance, such as stable Paretian laws. In addition, studying the performance of the considered distributions using weekly and monthly equity return data may produce different insights, just as weekly and monthly data may exhibit different characteristics than the daily data. Finally, another research idea is to extend our analysis on the behavior of distributions at the extreme values of each market index return using risk measures other than VaR. Despite being a widely used risk measure, VaR has been criticized for not properly presenting the full picture of the risks a company faces. In particular, a well-known shortcoming of VaR is that it is not a coherent risk measure (Artzner, Delbaen, Eber, \& Heath, 1999). An alternative coherent risk measure is the expected shortfall, also known as conditional VaR or tail loss. The extension of our analysis using the expected shortfall as the risk measure may yield additional insights.

\section{Appendix A.1. Plots obtained using the Kolmogorov-Smirnov test statistic}

This Appendix presents the computed Kolmogorov-Smirnov (KS) test statistic for developed and emerging markets considered in the paper under several distributions. Specifically, Fig. 1 presents the computed KS statistics for the developed markets of Australia, France, Japan, and Singapore; Fig. 2 presents the computed KS statistics for the developed markets of Spain, Switzerland, UK, and US; Fig. 3 presents the computed KS statistics for the emerging markets of Brazil, Chile, China, and India; and Fig. 4 presents the computed KS statistics for the emerging markets of Korea, Malaysia, Russia, and South Africa. In each figure, the KS statistic is compared with respect to the following six distributions: the skewed- $t$ distribution, the generalized lambda distribution (GLD), the normal inverse Gaussian (NIG) distribution, Johnson $S_{U}$ family, g-and-h distribution, and normal distribution.

\section{Appendix A.2. Plots obtained using the Anderson-Darling test statistic}

This Appendix presents the computed Anderson-Darling (AD) test statistic for developed and emerging markets considered in the paper under several distributions. Specifically, Fig. 5 presents the computed $\mathrm{AD}$ statistics for the developed markets of Australia, France, Japan, and Singapore; Fig. 6 presents the computed AD statistics for the developed markets of Spain, Switzerland, UK, and US; Fig. 7 presents the computed AD statistics for the emerging markets of Brazil, Chile, China, and India; and Fig. 8 presents the computed $\mathrm{AD}$ statistics for the emerging markets of Korea, Malaysia, Russia, and South Africa. In each figure, the AD statistic is compared with respect to the following five distributions: the 


\section{Australia}

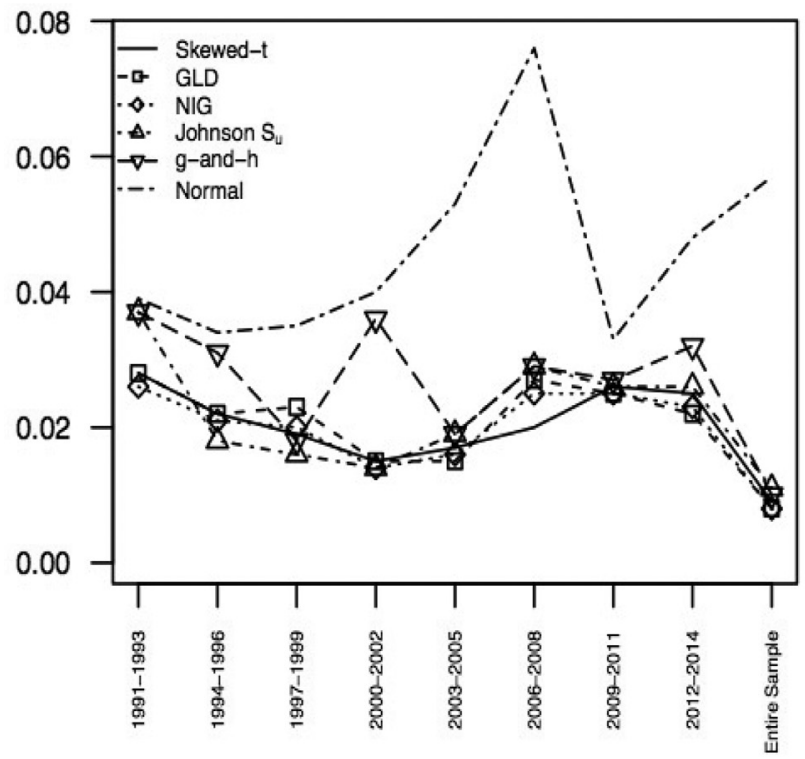

Japan

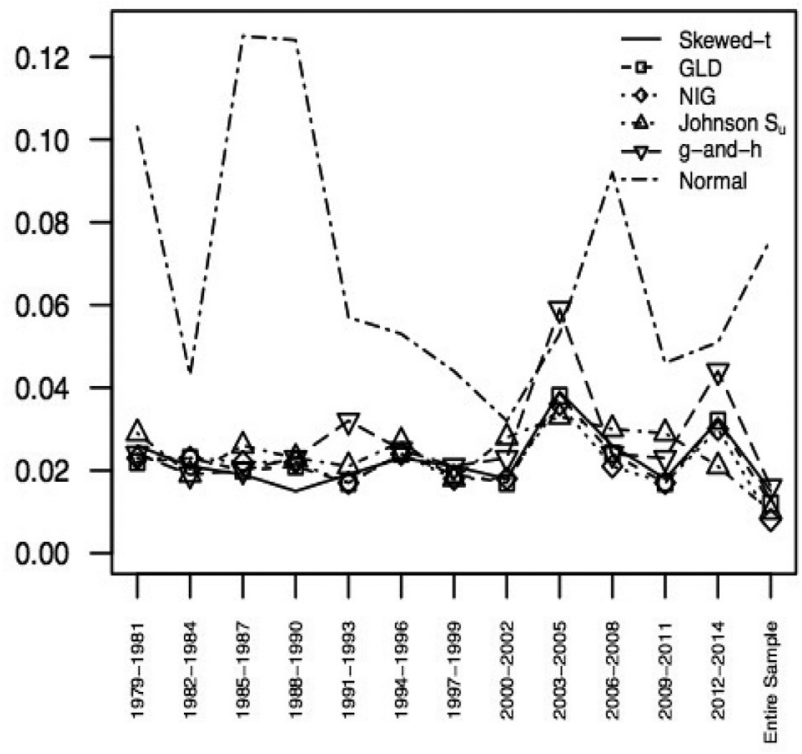

France

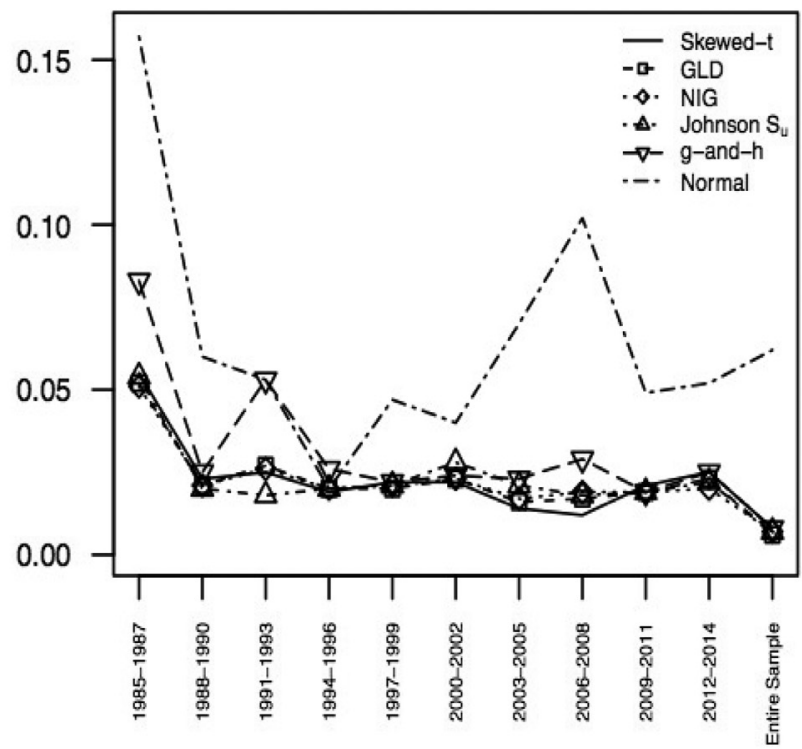

Singapore

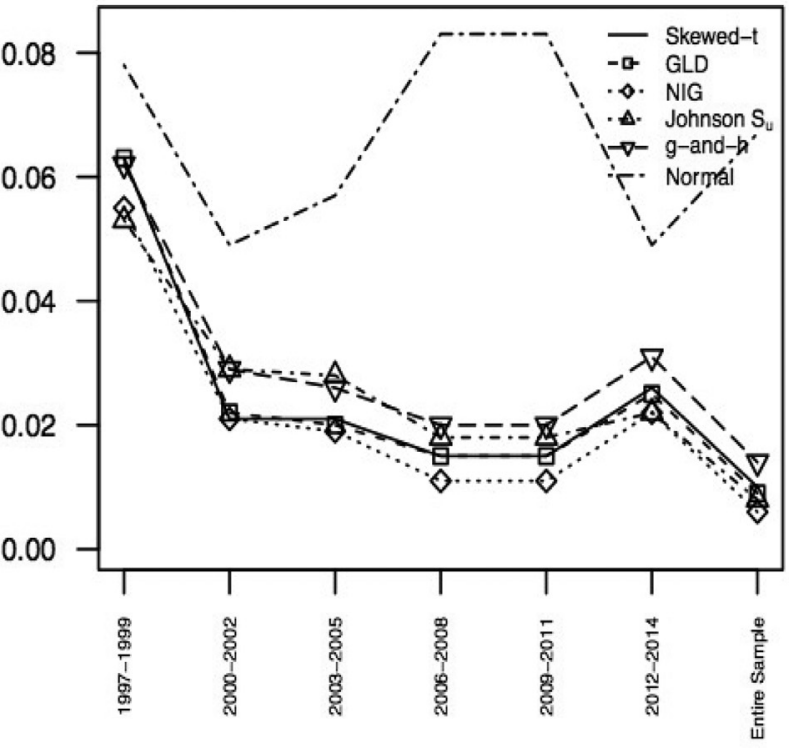

Fig. 1. KS statistics for the developed markets of Australia, France, Japan, and Singapore. 
Spain

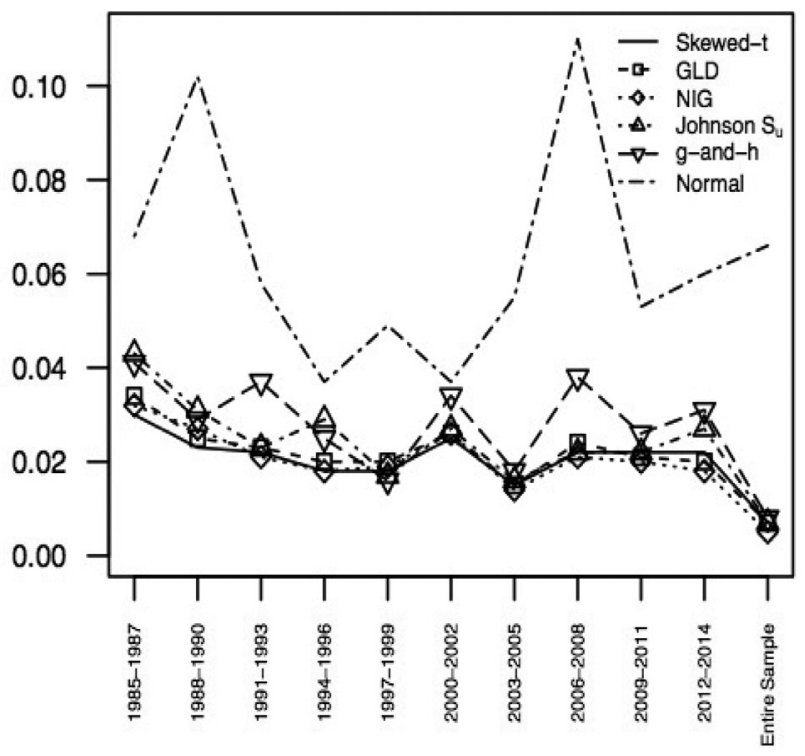

UK

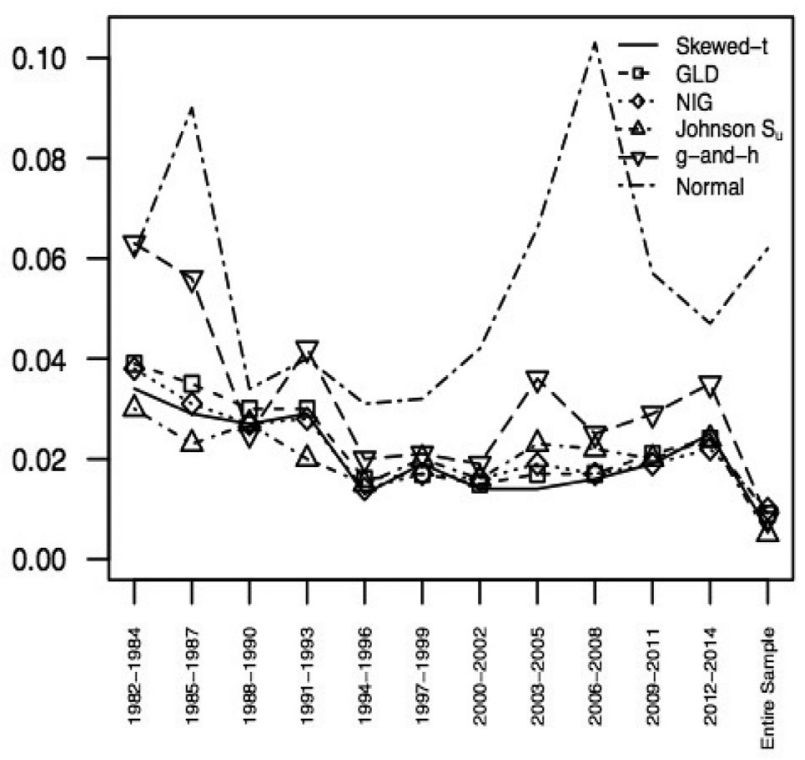

Switzerland

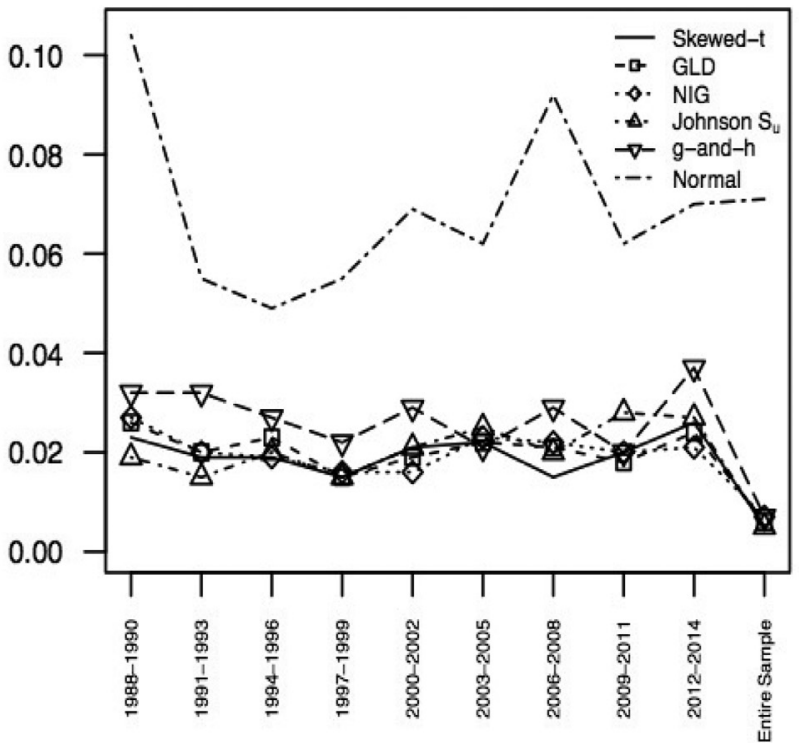

US

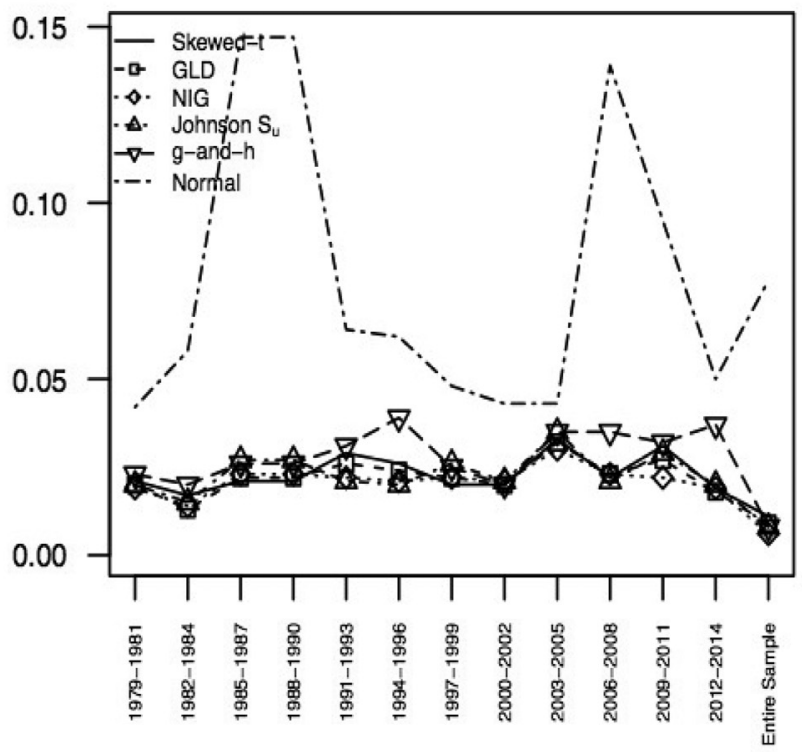

Fig. 2. KS statistics for the developed markets of Spain, Switzerland, UK, and US. 
Brazil

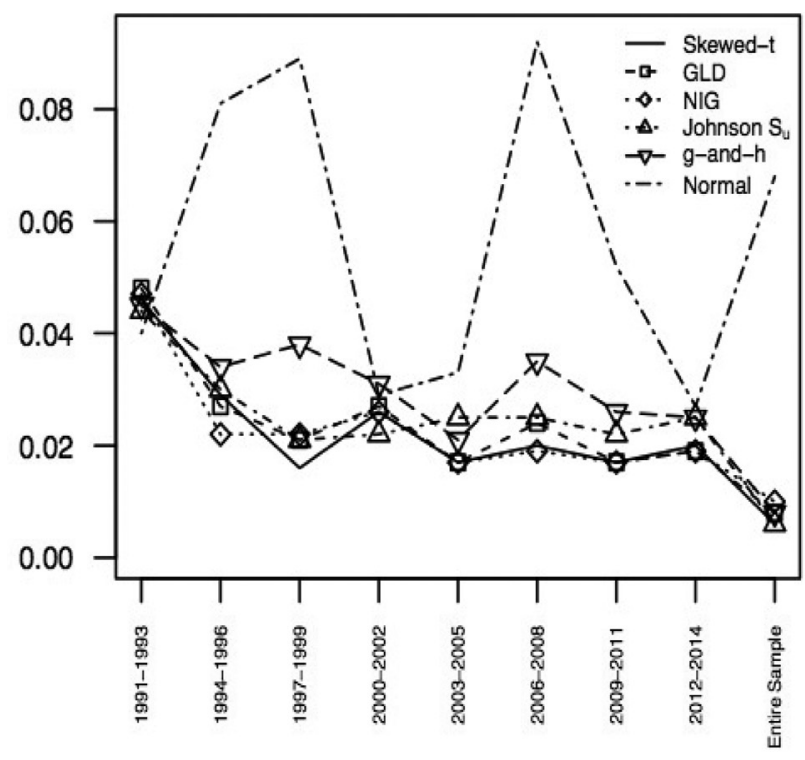

China

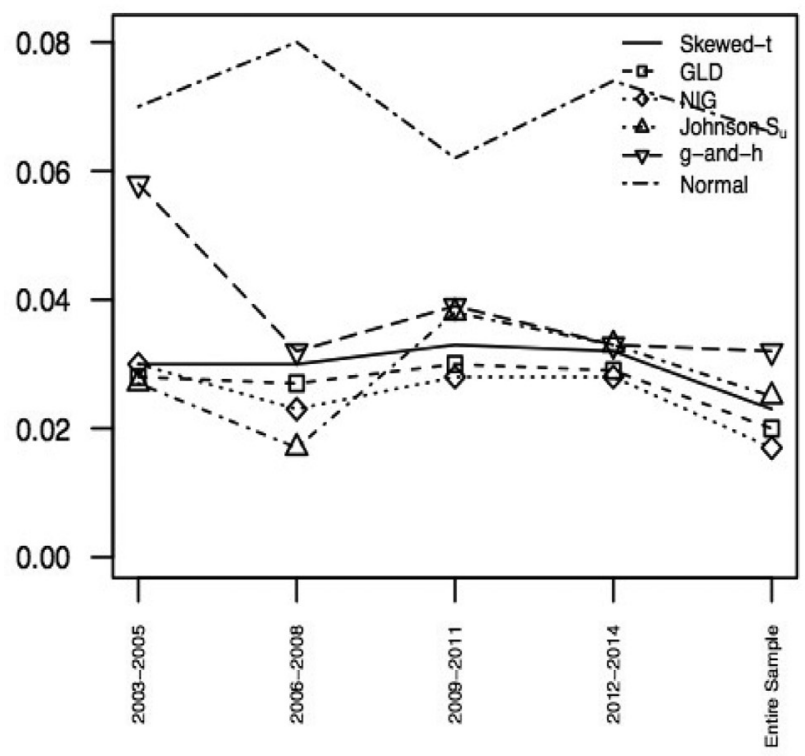

Chile

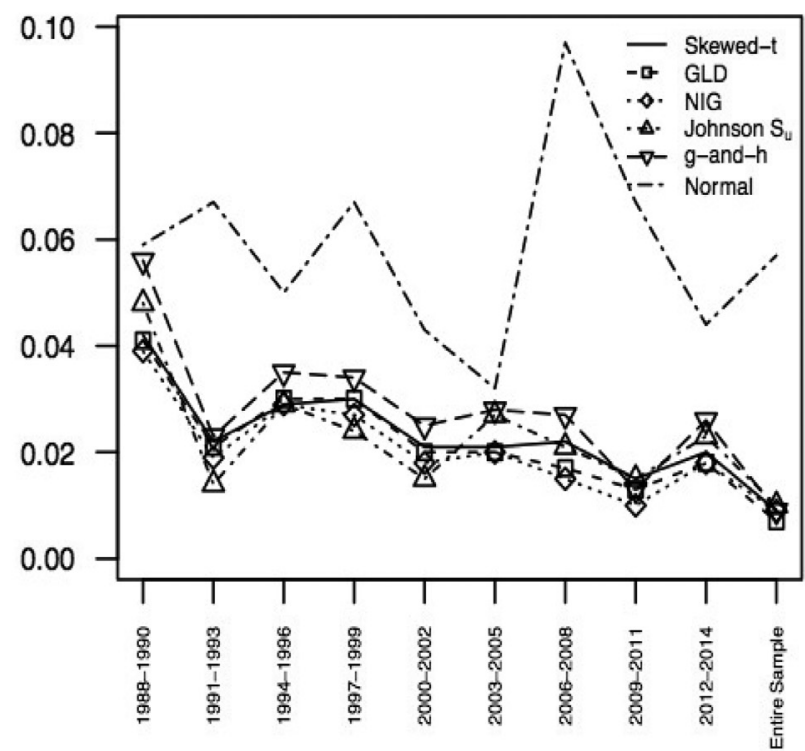

India

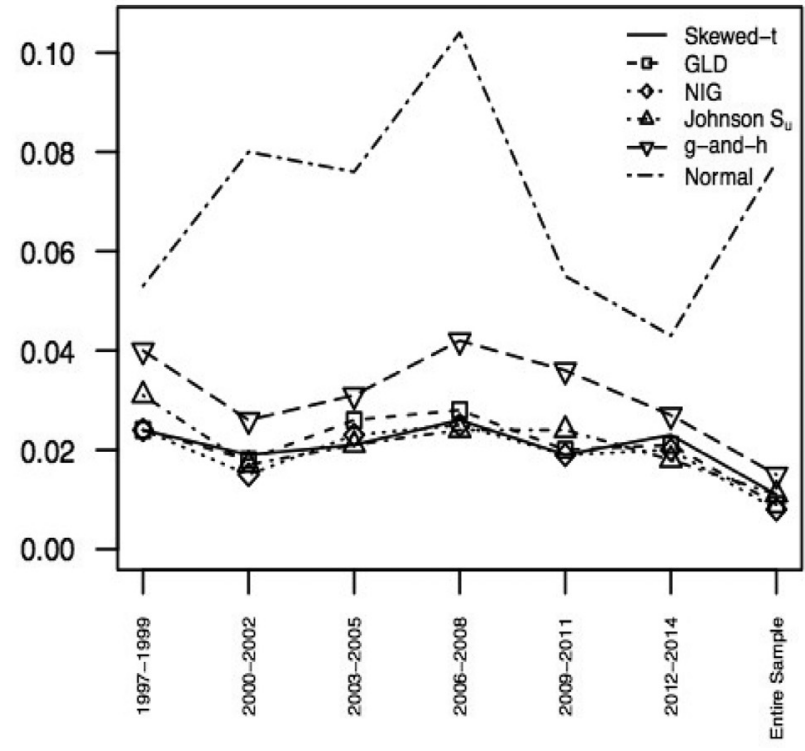

Fig. 3. KS statistics for the emerging markets of Brazil, Chile, China, and India. 
Korea

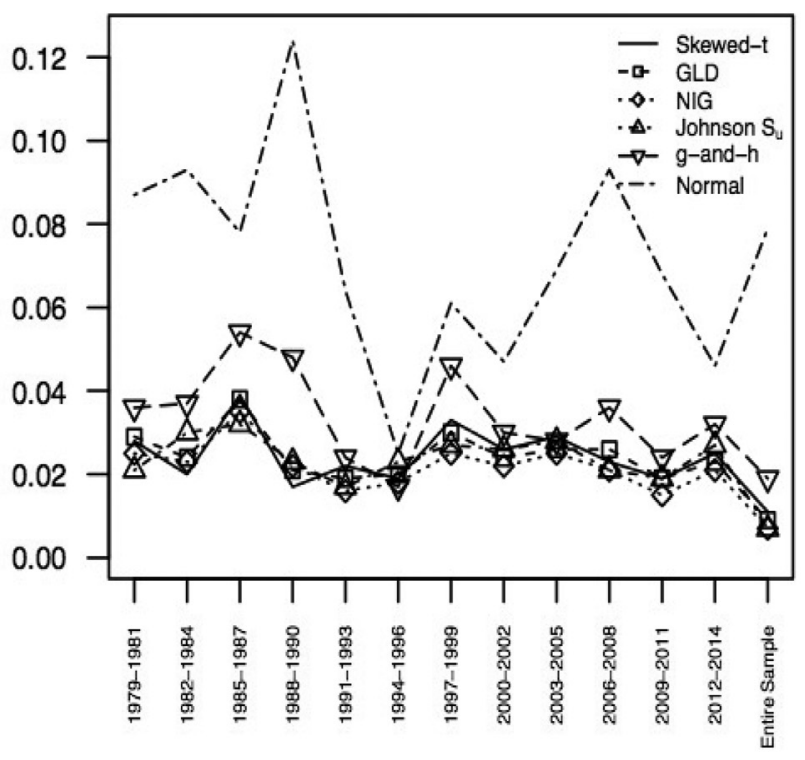

Russia

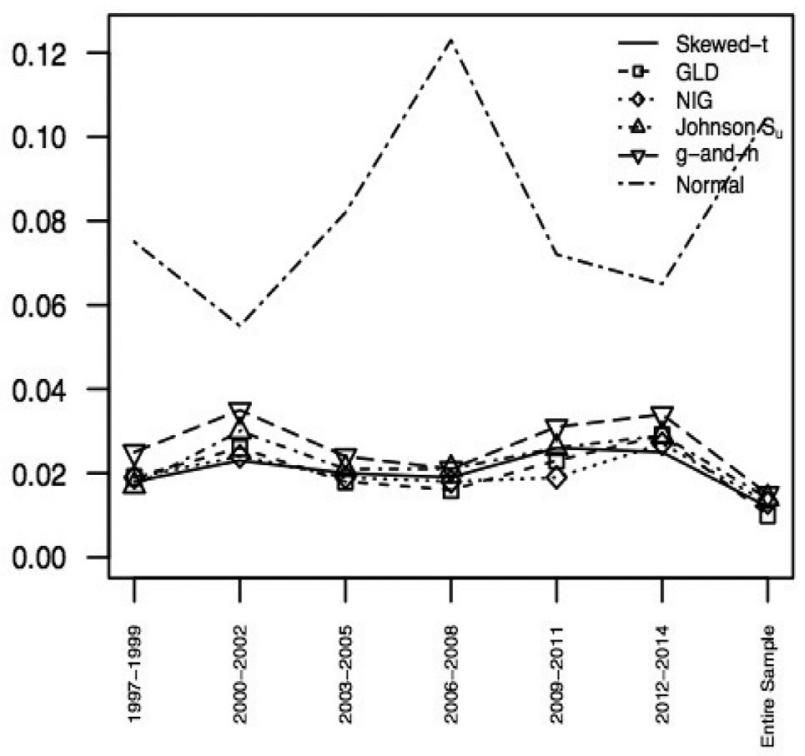

Malaysia

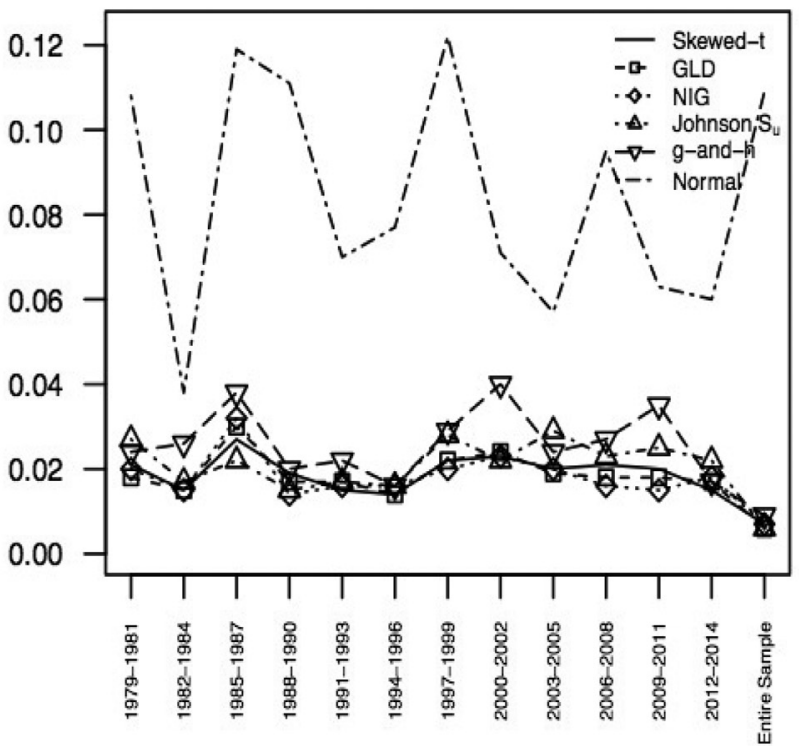

SouthAfrica

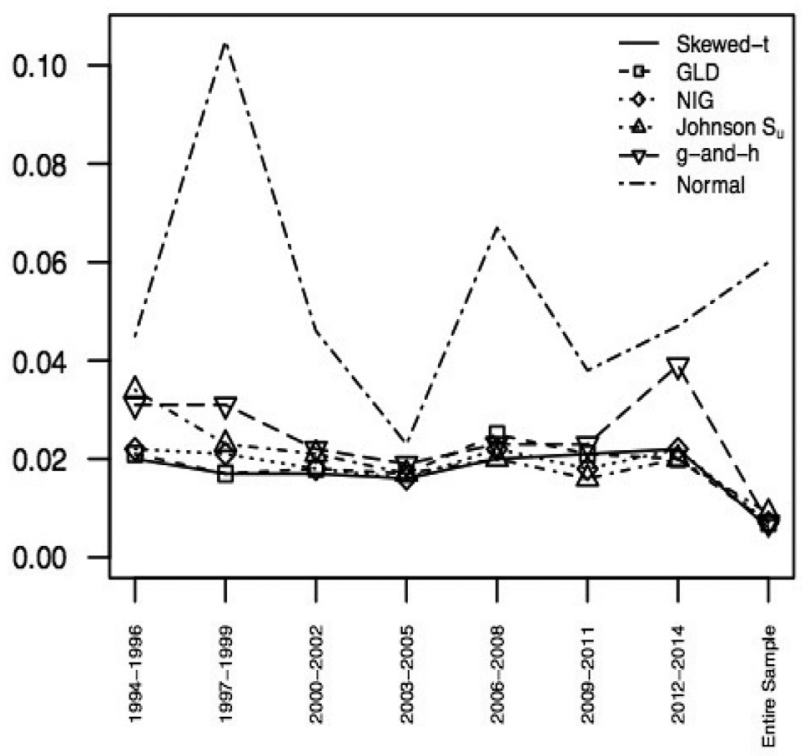

Fig. 4. KS statistics for the emerging markets of Korea, Malaysia, Russia, and South Africa. 
Australia

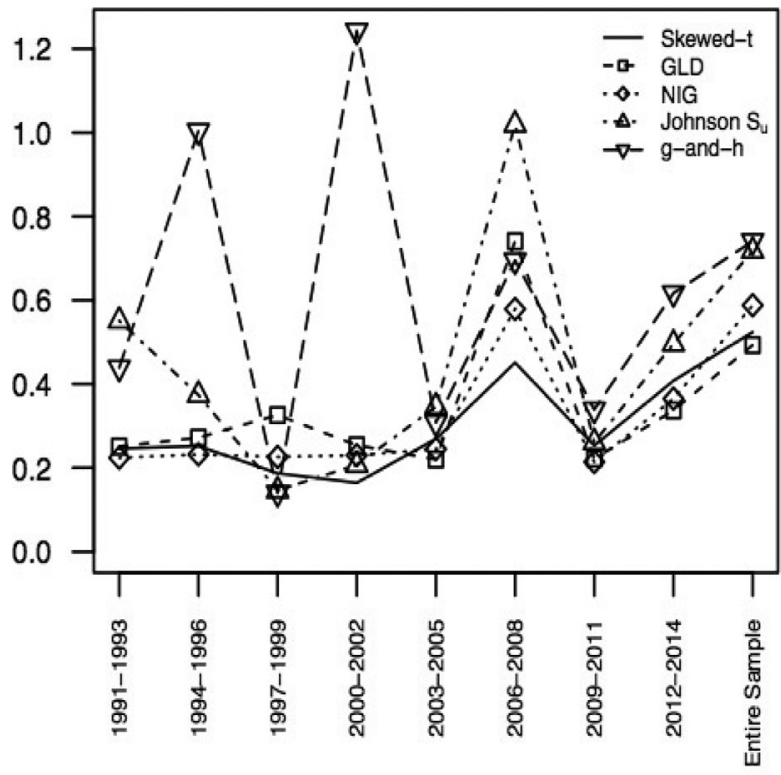

Japan

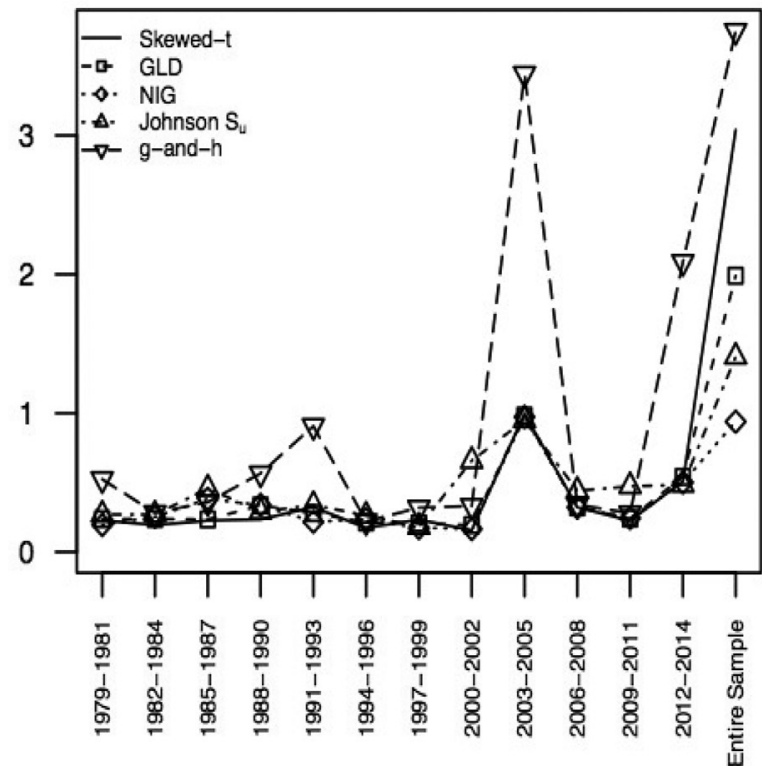

France

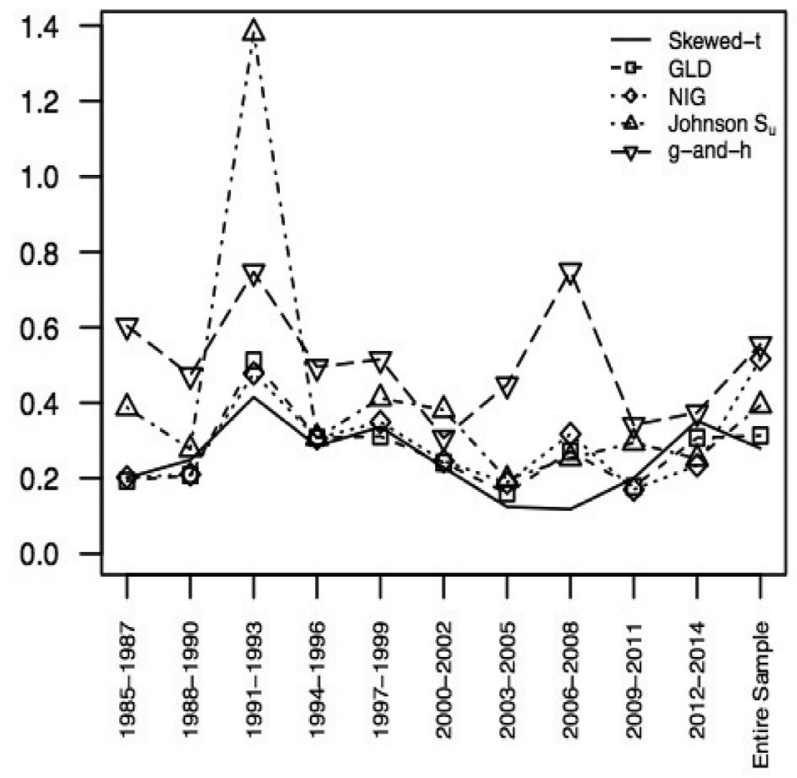

Fig. 5. AD statistics for the developed markets of Australia, France, Japan, and Singapore. 
Spain

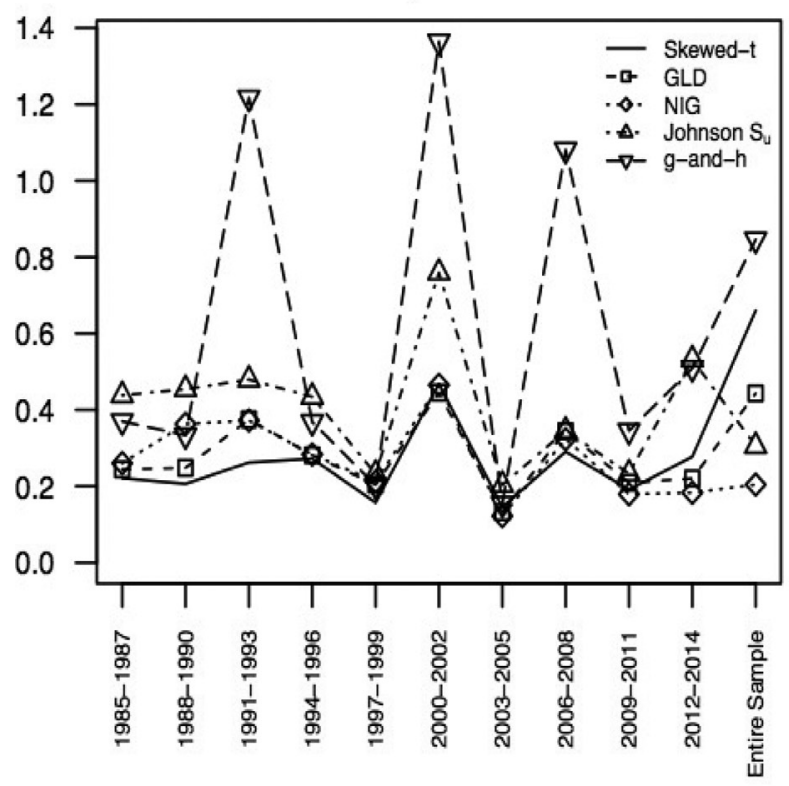

UK

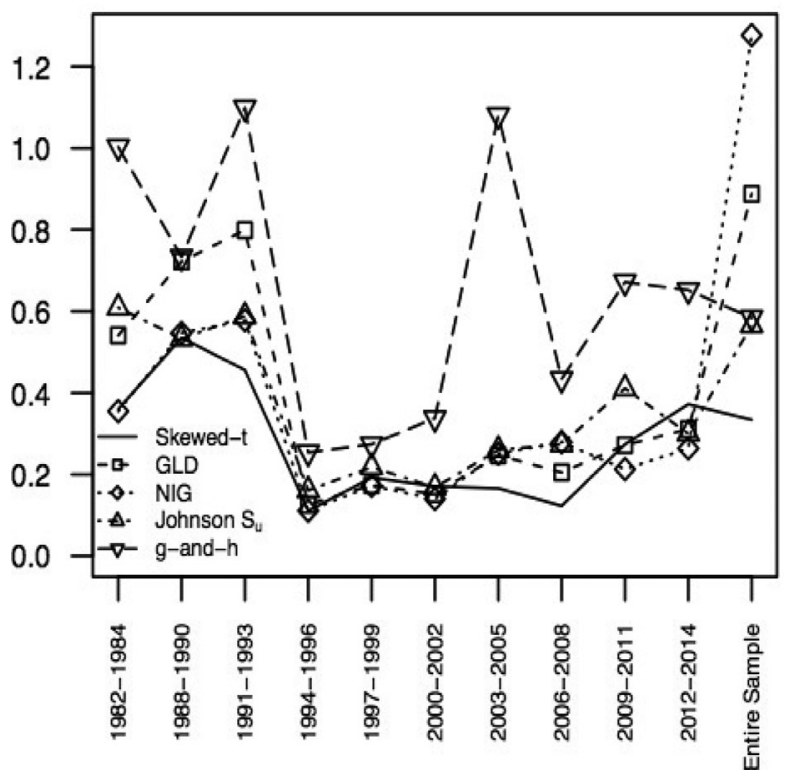

Switzerland

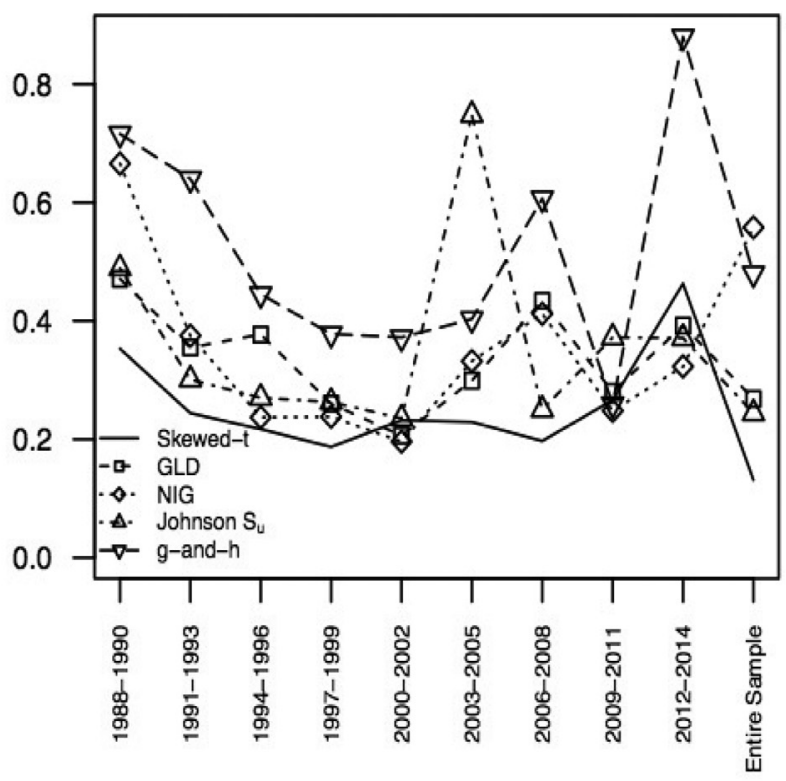

US

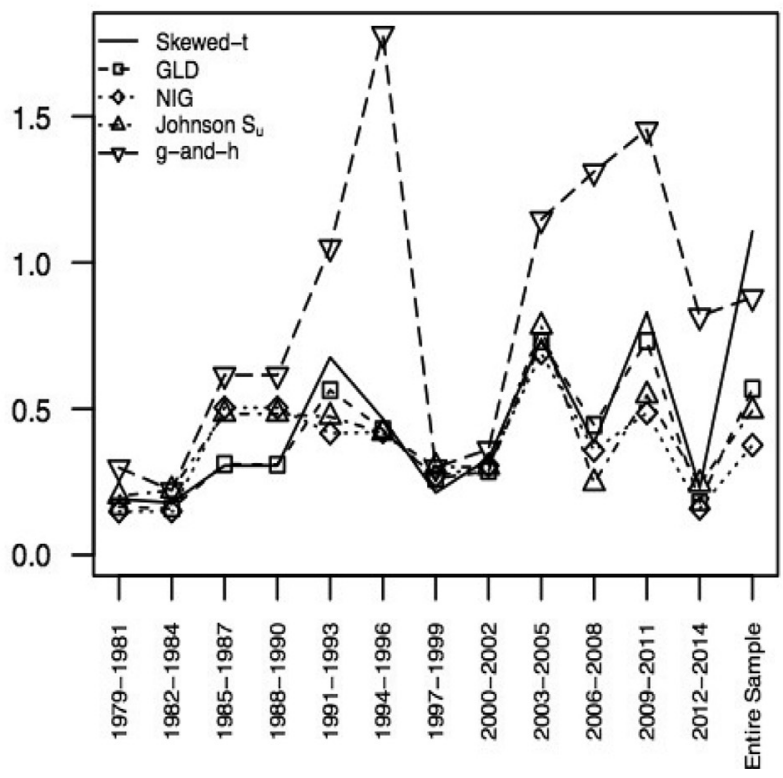

Fig. 6. AD statistics for the developed markets of Spain, Switzerland, UK, and US. 
Brazil

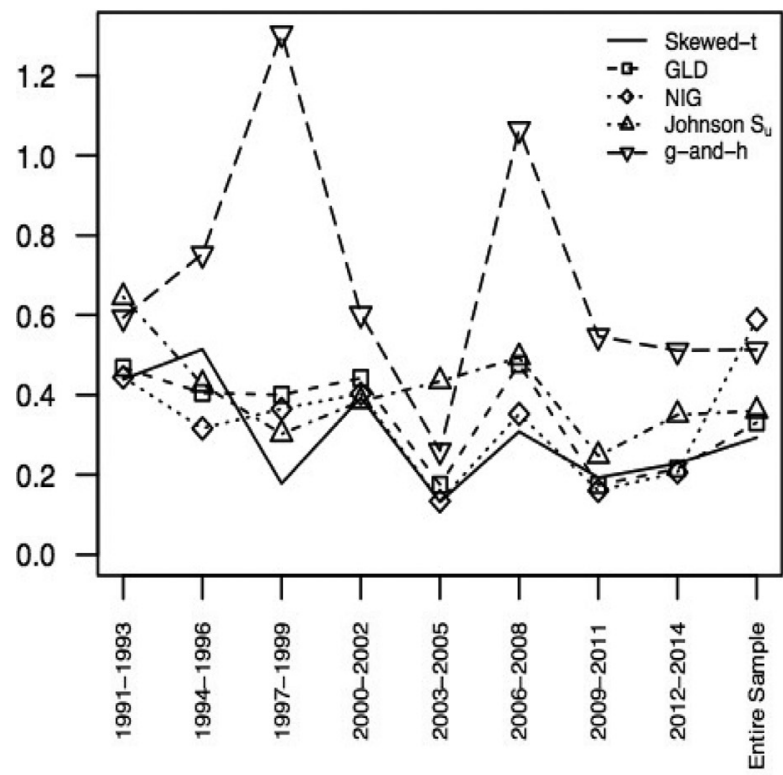

Chile

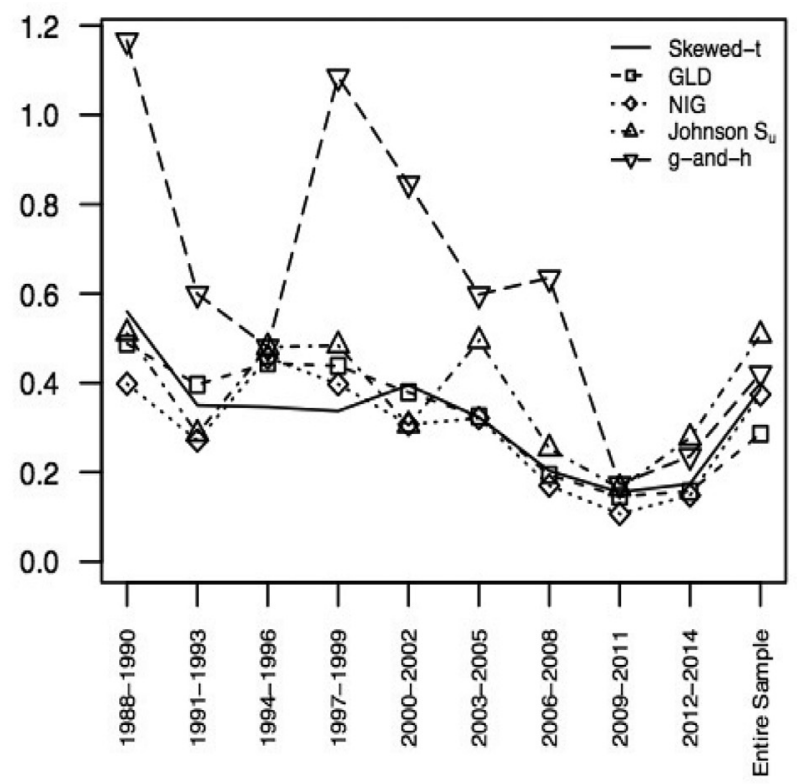

China

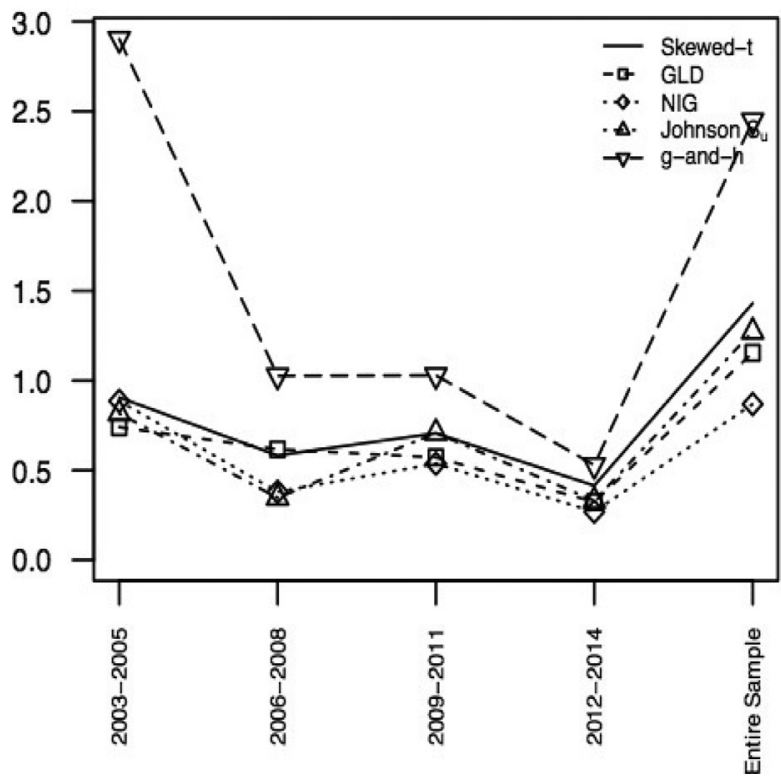

India

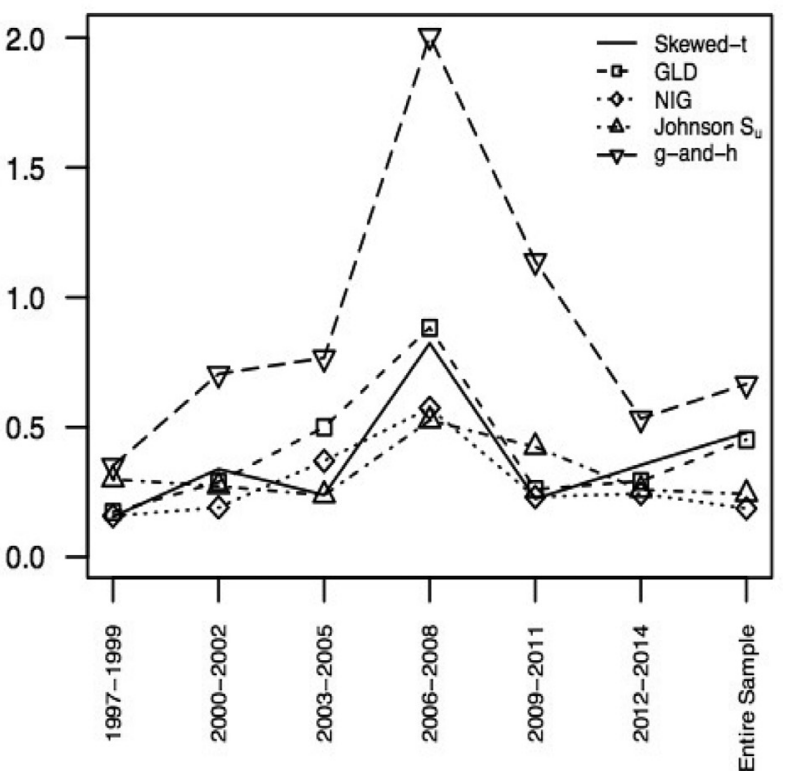

Fig. 7. AD statistics for the emerging markets of Brazil, Chile, China, and India. 
Korea

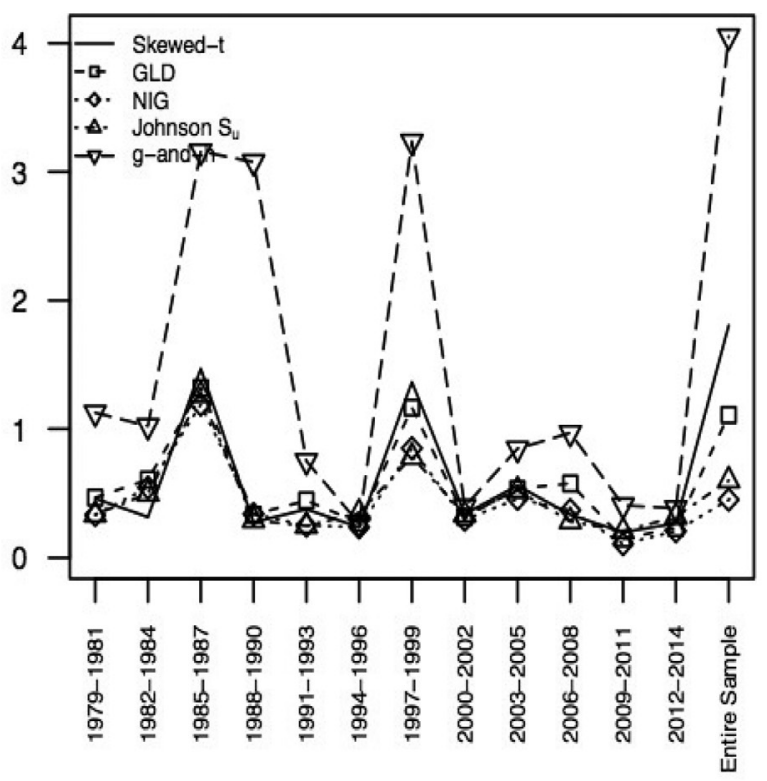

Russia

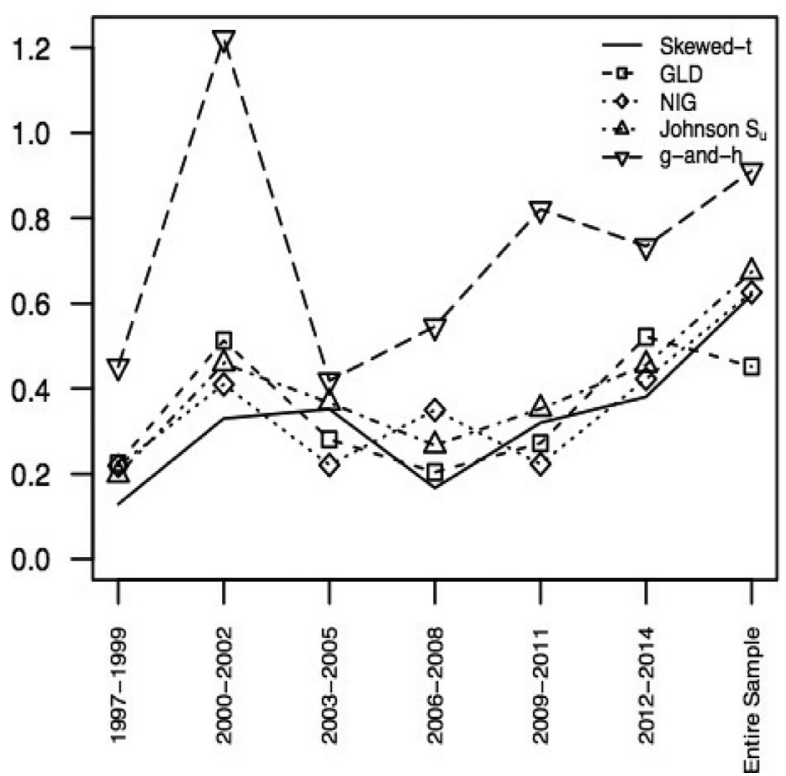

Malaysia

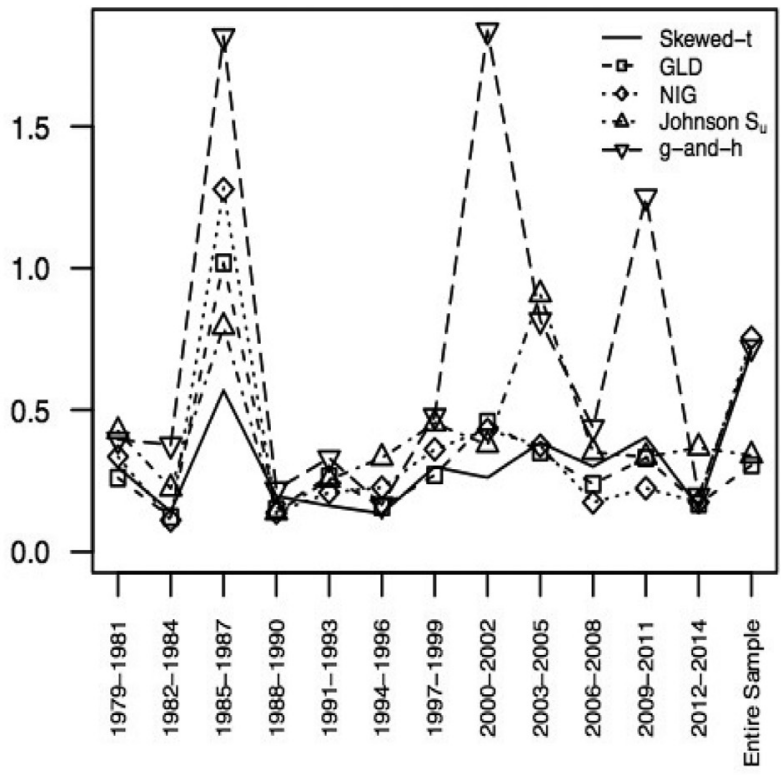

SouthAfrica

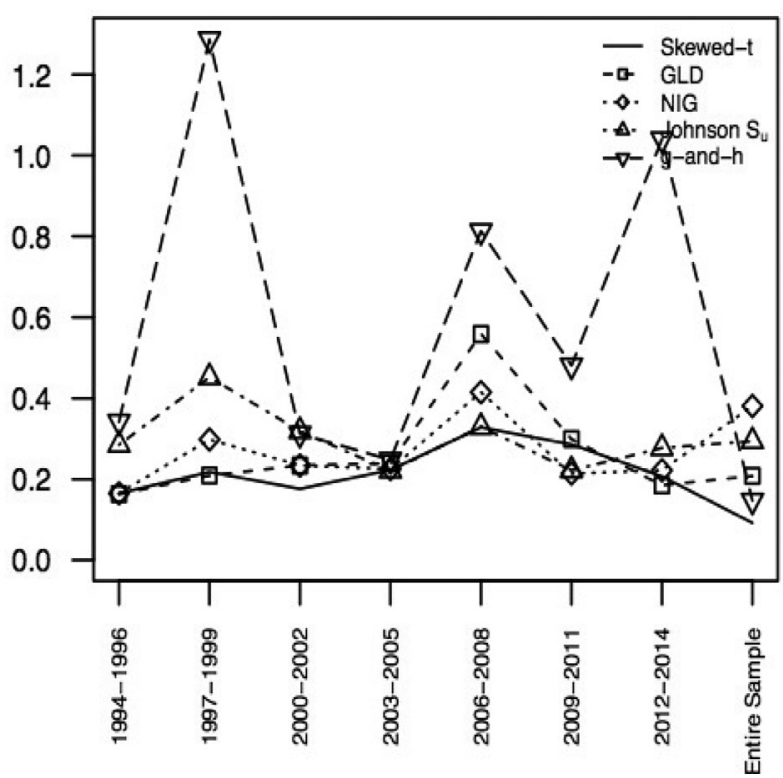

Fig. 8. AD statistics for the emerging markets of Korea, Malaysia, Russia, and South Africa. 
skewed- $t$ distribution, the generalized lambda distribution (GLD), the normal inverse Gaussian (NIG) distribution, Johnson $S_{U}$ family, and g-and-h distribution.

\section{References}

Akgiray, V., \& Booth, G. G. (1987). Compound distribution models of stock returns an empirical comparison. Journal of Financial Research, 10(3), 269-280.

Anderson, T. W., \& Darling, D. A. (1954). A test of goodness of fit. Journal of the American Statistical Association, 49(268), 765-769.

Aparicio, F. M., \& Estrada, J. (2001). Empirical distributions of stock returns: European securities markets, 1990-95. The European Journal of Finance, 7(1), 1-21.

Artzner, P., Delbaen, F., Eber, J.-M., \& Heath, D. (1999). Coherent measures of risk. Mathematical Finance, 9(3), 203-228.

Azzalini, A., \& Capitanio, A. (2003). Distributions generated by perturbation of symmetry with emphasis on a multivariate skew t-distribution. Journal of the Royal Statistical Society: Series B (Statistical Methodology), 65(2), 367-389.

Bachelier, L. (1900). Théorie de la spéculation. In Annales scientifiques de l'école normale supérieure: Vol. 17 (pp. 21-86). Société mathématique de France.

Badrinath, S., \& Chatterjee, S. (1988). On measuring skewness and elongation in common stock return distributions: The case of the market index. The Journal of Business, 61(4), 451-472.

Behr, A., \& Pötter, U. (2009). Alternatives to the normal model of stock returns: Gaussian mixture, generalised $\log \mathrm{F}$ and generalised hyperbolic models. Annals of Finance, 5(1), 49-68.

Blattberg, R. C., \& Gonedes, N. J. (1974). A comparison of the stable and Student distributions as statistical models for stock prices. The Journal of Business, 47(2), 244-280.

Bølviken, E., \& Benth, F. E. (2000). Quantification of risk in Norwegian stocks via the normal inverse Gaussian distribution. In Afir 2000 colloquim, Tromsø, Norway (pp. 87-98)

Bookstaber, R. M., \& McDonald, J. B. (1987). A general distribution for describing security price returns. The Journal of Business, 60(3), 401-424.

Chakravarti, I., \& Laha, R. (1967). Handbook of methods of applied statistics: Vol. 1. John Wiley \& Sons.

Chalabi, Y., Scott, D. J., \& Würtz, D. (2010). The generalized Lambda distribution as an alternative to model financial returns. Technical report. Eidgenössische Technische Hochschule and University of Auckland, Zurich and Auckland.

Corlu, C. G., \& Biller, B. (2015). Least-squares and Bayesian inferences for Johnson's $S_{L}$ and $\mathrm{S}_{\mathrm{B}}$ distributions, Metropolitan College, Boston University, Boston, MA.Working paper.

Corlu, C. G., \& Meterelliyoz, M. (2015). Estimating the parameters of the generalized lambda distribution: Which method performs best? Communications in Statistics - Simulation and Computation.. doi:10.1080/03610918.2014.901355.

Dutta, K. K., \& Babbel, D. F. (2002). On measuring skewness and kurtosis in short rate distributions: The case of the US Dollar London inter bank offer rates. Technical report. Wharton School Center for Financial Institutions, University of Pennsylvania.

Eberlein, E., \& Keller, U. (1995). Hyperbolic distributions in finance. Bernoulli, 1(3), 281-299.

Fama, E. F. (1963). Mandelbrot and the stable Paretian hypothesis. The Journal of Business, 36(4), 420-429.

Fama, E. F. (1965). The behavior of stock-market prices. Journal of Business, 38(1), 34-105.

Fernandez, P., Aguirreamalloa, J., \& Avendaño, L. C. (2011). Ibex 35: 1991-2010 value creation and return. Http://ssrn.com/abstract=1737860. Accessed 5.11.15.

Filliben, J. J. (1975). The probability plot correlation coefficient test for normality. Technometrics, 17(1), 111-117.

Fournier, B., Rupin, N., Bigerelle, M., Najjar, D., Iost, A., \& Wilcox, R. (2007). Estimating the parameters of a generalized lambda distribution. Computational Statistics E' Data Analysis, 51(6), 2813-2835.

Freimer, M., Kollia, G., Mudholkar, G. S., \& Lin, C. T. (1988). A study of the generalized Tukey lambda family. Communications in Statistics-Theory and Methods, 17(10), 3547-3567.

Gray, J. B., \& French, D. W. (1990). Empirical comparisons of distributional models for stock index returns. Journal of Business Finance E' Accounting, 17(3), 451-459.
Hagerman, R. L. (1978). More evidence on the distribution of security returns. The Journal of Finance, 33(4), 1213-1221.

Harris, R. D. \& Küçüközmen, C. C. (2001a). The empirical distribution of stock returns: Evidence from an emerging European market. Applied Economics Letters, 8(6), 367-371.

Harris, R. D., \& Küçüközmen, C. C. (2001b). The empirical distribution of UK and US stock returns. Journal of Business Finance \& Accounting, 28(5-6), 715-740.

Hastings, C., Mosteller, F., Tukey, J. W., \& Winsor, C. P. (1947). Low moments for small samples: A comparative study of order statistics. The Annals of Mathematical Statistics, 18(3), 413-426.

Hoaglin, D. C. (2006). Summarizing shape numerically: The g-and-h distributions. In Exploring Data Tables, Trends, and Shapes (pp. 461-513). John Wiley \& Sons, Inc.

Hsu, D.-A., Miller, R. B., \& Wichern, D. W. (1974). On the stable Paretian behavior of stock-market prices. Journal of the American Statistical Association, 69(345), 108113.

Johnson, N. L. (1949). Systems of frequency curves generated by methods of translation. Biometrika, 36(1/2), 149-176.

Joiner, B. L., \& Rosenblatt, J. R. (1971). Some properties of the range in samples from Tukey's symmetric lambda distributions. Journal of the American Statistical Association, 66(334), 394-399.

Kon, S. J. (1984). Models of stock returns-a comparison. The Journal of Finance, 39(1), 147-165.

Kupiec, P. H. (1995). Techniques for verifying the accuracy of risk measurement models. The Journal of Derivatives, 3(2), 73-84.

Lau, A. H.-L., Lau, H.-S., \& Wingender, J. R. (1990). The distribution of stock returns: New evidence against the stable model. Journal of Business \& Economic Statistics, $8(2), 217-223$.

Linden, M. (2001). A model for stock return distribution. International Journal of Finance \& Economics, 6(2), 159-169.

Mandelbrot, B. (1967). The variation of some other speculative prices. Journal of Business, 40(4), 393-413.

Mills, T. C. (1995). Modelling skewness and kurtosis in the London Stock Exchange FT-SE index return distributions. Journal of the Royal Statistical Society. Series D (The Statistician), 44(3), 323-332.

Officer, R. R. (1972). The distribution of stock returns. Journal of the American Statistical Association, 67(340), 807-812.

Osborne, M. F. (1959). Brownian motion in the stock market. Operations Research, $7(2), 145-173$

Pfaff, B., McNeil, A., \& Ulmann, S. (2013). Qrm: Provides R-language code to examine quantitative risk management concepts. $R$ package version 0.4-9.URL http: //CRAN.R-project.org/package=QRM. Accessed 5.11.15.

Praetz, P. D. (1972). The distribution of share price changes. Journal of Business, 45(1), 49-55.

Prause, K. (1999). How to use NIG laws to measure market risk. FDM Preprint 65 University of Freiburg.

Prause, K., Zentrum, F., \& Modellbildung, D. (1997). Modelling financial data using generalized hyperbolic distributions. FDM Preprint 48 Technical Report. University of Freiburg.

Rachev, S. T., \& Mittnik, S. (2000). Stable Paretian models in finance. Chichester, UK: John Wiley \& Sons.

Rachev, S. T., Stoyanov, S. V., Biglova, A., \& Fabozzi, F. J. (2005). An empirical examination of daily stock return distributions for US stocks. In D. Baier, R. Decker, \& L. Schmidt-Thieme (Eds.), Data analysis and decision support. In Series in Studies in Classification, Data Analysis, and Knowledge Organization (pp. 269-281). Springer Berlin Heidelberg.

Ramberg, J. S., \& Schmeiser, B. W. (1974). An approximate method for generating asymmetric random variables. Communications of the ACM, 17(2), 78-82.

Ross, S. M. (2004). Simulation (4th ed.). Orlando, FL, USA: Academic Press, Inc.

Shilling, H. (1996). International guide to securities market indices. Florida, USA: CRC Press.

Su, S. (2007). Numerical maximum log likelihood estimation for generalized lambda distributions. Computational Statistics \& Data Analysis, 51(8), 3983-3998.

Tuenter, H. J. (2001). An algorithm to determine the parameters of $S_{U}$-curves in the Johnson system of probability distributions by moment matching. Journal of Statistical Computation and Simulation, 70(4), 325-347.

Tukey, J. (1977). Exploratory data analysis. Addison-Wesley series in behavioral science. Addison-Wesley Publishing Company. 